\title{
A high-order streamline Godunov scheme for steady supersonic/hypersonic equilibrium flows
}

\author{
Jaw-Yen Yang ${ }^{a, *}$, Yun-Hai Tang ${ }^{\mathrm{b}, 1}$, Shih-Tuen Lee ${ }^{\mathrm{b}, 2}$ \\ ${ }^{2}$ Institute of Applied Mechanics, National Taiwan University, Taipei, Taiwan, ROC \\ ${ }^{\mathrm{b}}$ Department of Mechanical Engineering, National Taiwan University, Taipei, Taiwan, ROC
}

Received 11 April 1997; accepted 7 August 1997

\begin{abstract}
A space marching Godunov-type method using control volumes consisted of streamlines and the coordinate lines in the marching direction is described for the computation of steady supersonic/hypersonic equilibrium flows. A steady Riemann problem and its solution for equilibrium gases is described and used in the Godunov method. Both Tannehill's equilibrium air program and Lighthill's ideal dissociating gas model are used to calculate the equilibrium gas properties. The streamline meshes are automatically solution gerterated. The use of streamline meshes enables a crisp resolution of sliplines and simplifies the Godunov solution procedure. Extensive computations of various steady two-dimensional and three-dimensional axisymmetrical supersonic/hypersonic equilibrium flows are included to illustrate the method. (C) 1998 Elsevier Science S.A.
\end{abstract}

\section{Introduction}

In [1], we have described a streamline Godunov-type scheme for the computation of steady supersonic and hypersonic flows of perfect gases. In this sequel, we shall describe the same numerical approach for the computation of steady supersonic/hypersonic equilibrium flows. In order to implement the Godunov method [2], one has to find the solution for the steady Ricmann problem for an equilibrium gas. One of the most important tasks one has to deal with in order to obtain accurate and efficient numerical solutions of inviscid equilibrium flows is to be able to obtain the accurate equilibrium gas properties. Since the equation of state for an equilibrium real gas is not available in closed form, it must either be obtained by solving a set of nonlinear chemical equilibrium equations, or approximated by some curve fit or a table lookup. In recent years formulations for various approximate Riemann solver for the Euler equations for equilibrium gases have been reported [3-8]. Here, we use the Tannehill's equilibrium air program [9] and Lighthill's ideal dissociating gas model [10] to calculate the real gas properties. In [9], the equilibrium gas properties such as pressure, temperature and speed of sound, as functions of density and internal energy, can be efficiently calculated. This program is simple and efficient and thus is very suitable for modern computational fluid dynamics, particularly for high-speed flows. In [10], Lighthill proposed an interesting and simplified model called the ideal dissociating gas (IDG) for calculating the equilibrium properties of gases. In the IDG model to obtain all the equilibrium gas properties one has to use the numerical iteration for the law of mass action and the equation of state, although

\footnotetext{
* Corresponding author. Professor, Institute of Applied Mechanics.

' Graduate Student, Department of Mechanical Engineering.

${ }^{2}$ Professor, Department of Mechanical Engineering.
} 
the formula for an equilibrium gas is explicit and easy to understand. Although this model has proved useful in the approximate study of dissociating symmetrical diatomic gas, however, it has not been broadly used in modern computational gas dynamics. Here, since air before dissociation is a mixture primarily consisting of $\mathrm{O}_{2}$ and $\mathrm{N}_{2}$, in this connection we also consider this IDG model. A more recent program for equilibrium air has been provided by Vinokur and Liu [11]. Although this program, in principle, can also be adopted in the present frame of work, however, due to the different formulation and independent variables used and to limit the size of the paper we shall not include this equilibrium real gas model in this work. Other general equilibrium gas laws applied to numerical formulations for various approximate Riemann solvers including the Steger-Warming flux vector splitting [12], the van Leer flux vector splitting [13], and Roe's approximate Riemann solver [14], have been given.

The purpose of the present work is to construct an efficient and second-order Godunov-type space marching scheme for steady supersonic/hypersonic equilibrium flow computations based on the steady Riemann problem and its solution for an equilibrium gas using streamline meshes. As a building block, the Riemann problem and its solution play an essential role in the Godunov-type scheme in the numerical solution of inviscid compressible gas flows. For perfect gases, exact Riemann solution for steady Euler equations can be found in [15]. The solution procedure for real gases is similar to that for the perfect gases. However, due to the different real gas models implemented, the detailed steps can be quite different. Here, two real gas models are used. One is due to Tannehill and the other one Lighthill. In this work, pressure is used as the independent thermodynamic variable in order to meet the condition of pressure equality at both sides of slipline and the real gas properties are calculated due to Srinivasan et. al. [9] and Vincenti and Kruger [16] can also be used.

The characteristics of using streamline meshes as control volumes or cells in the Godunov method has been illustrated in [1]. Basically, the use of streamline meshes shares the same desirable features of new Lagrangian method [17] via simplifying the Godunov solution procedure and producing crisp resolution of the sliplines since for steady flow a slipline coincides a streamline, just as in [17]. To improve the accuracy of the basic Godunov method which is first-order accurate we extend it to second-order method $[18,19]$ by adopting an essentially nonoscillatory interpolation due to Harten and Osher [20] (see also [1]).

In Section 2, the steady Euler equations of inviscid compressible equilibrium flows are described. In Section 3 , the streamline Godunov-type scheme of second-order accuracy is briefly outlined. The elementary waves of the steady Riemann problem for an equilibrium gas and its solutions are given in Section 4. Formulations are derived using pressure as independent thermodynamic variable. In Section 5, 'Tannehill's air table and Lighthill's ideal dissociation gas model used are introduced. In Section 6, numerical results for various steady supersonic and hypersonic equilibrium flow problems are given to illustrate the feature of the present method. Some concluding remarks are made in Section 7.

\section{Steady Euler equations of real gas}

The Euler equations of motion of an inviscid non-heat-conducting gas for steady two-dimensional and three-dimensional axisymmetrical equilibrium flows in conservation form can be written as

$$
\frac{\partial \boldsymbol{E}}{\partial x}+\frac{\partial \boldsymbol{F}}{\partial y}+\boldsymbol{S}=\mathbf{0}
$$

where

$$
\boldsymbol{E}=\left(\begin{array}{c}
\rho u \\
\rho u^{2}+P \\
\rho u v \\
\rho u H
\end{array}\right)=\left(\begin{array}{c}
\mathrm{E}_{1} \\
\mathrm{E}_{2} \\
\mathrm{E}_{3} \\
\mathrm{E}_{4}
\end{array}\right), \quad \boldsymbol{F}=\left(\begin{array}{c}
\rho v \\
\rho u v \\
\rho v^{2}+P \\
\rho v H
\end{array}\right), \quad \boldsymbol{S}=\frac{\aleph}{y}\left(\begin{array}{c}
\rho v \\
\rho u v \\
\rho v^{2} \\
\rho v H
\end{array}\right) .
$$

Here, $x$ and $y$ are the physical coordinates, $\rho$ is the density of the gas, $u$ and $v$ are the corresponding components of the local velocity $V, P$ is the pressure, and $H$ is the total enthalpy per unit mass. The value of $N$ is 0 for the two-dimensional case and 1 for the axisymmetrical onc. In the later casc, $y$ denotes the radial 
coordinate, while $x$ denotes the space-marching direction in both cases. The relations among the state variables for equilibrium gases are further defined by two equations of state (EOS) which are taken in the form

$$
P=P(\rho, T) ; \quad h=h(\rho, T),
$$

where $T$ is the temperature and $h$ is the specific enthalpy. The total enthalpy per unit mass $H$ is

$$
H=h(\rho, P)+\frac{V^{2}}{2}=e(\rho, P)+\frac{P}{\rho}+\frac{V^{2}}{2},
$$

where $V^{2}=u^{2}+v^{2}$ and $e$ is the specific internal energy. For an inviscid non-heat-conducting flow, $H$ always conserves along the same streamline.

With the state variable vector $\boldsymbol{Q}=(\rho, u, v, P)^{\top}$, the matrix $[\partial \boldsymbol{E} / \partial \boldsymbol{Q}]^{-1}[\partial \boldsymbol{F} / \partial \boldsymbol{Q}]$, has four eigenvalues:

$$
\begin{aligned}
& \lambda_{0}=-\frac{v}{u} \text { multiplicity of } 2, \\
& \lambda_{ \pm}=\frac{u v \pm a \sqrt{V^{2}-a^{2}}}{u^{2}-a^{2}},
\end{aligned}
$$

where $a$ is the speed of sound. To guarantee that $[\partial E / \partial Q]^{-1}[\partial F / \partial Q]$ has four real eigenvalues, we require $V=\sqrt{u^{2}+v^{2}}>a$. That is, the flow must be supersonic. Then, the system (1) is hyperbolic and the spatial variable $x$ is a time-like variable. It is well known that 2-D steady supersonic flow and 1-D unsteady flow have the same mathematical structure and some qualitative analogy in flowfield, because both belong to the class of hyperbolic partial differential equations with two independent variables. Due to the similarity in mathematical structure and flowfield physics, we can obtain the solutions of these two kinds of problems by applying the same numerical method. Here, a marching method in the $x$-direction can be constructed to solve Eq. (1), and consider a space-marching method associated with the Godunov scheme. The numerical solution of Eq. (1) by Godunov-type scheme and a second-order essentially nonoscillatory (ENO2) interpolation has been described in detail in [1] for a perfect gas.

To solve the governing equations with the source terms, like Eq. (1), we can apply the splitting technique in our problem. That is, in the case of axisymmetrical flow, the numerical integrations for the source terms can be treated separately by using an appropriate $x$-marching step. When $\boldsymbol{E}^{n}$ represent finite difference approximations to the convection fluxes $\boldsymbol{E}$ at $x=n \Delta x$, the present computational scheme can be written as

$$
\boldsymbol{E}^{n+1}=\mathscr{L}^{S}(\Delta x / 2) \mathscr{L}^{F}(\Delta x) \mathscr{L}^{S}(\Delta x / 2) \boldsymbol{E}^{n} .
$$

Here, the operator $\mathscr{L}^{S}$ represents the inlegration of source terms, satisfying

$$
\frac{\partial \boldsymbol{E}}{\partial x}+\boldsymbol{S}=\mathbf{0}
$$

and the operator $\mathscr{L}^{\boldsymbol{F}}$ denotes the finite-volume integration of equation satisfying

$$
\frac{\partial \boldsymbol{E}}{\partial x}+\frac{\partial \boldsymbol{F}}{\partial y}=\mathbf{0} \text {. }
$$

The operator $\mathscr{L}^{S}$ is defined by

$$
\mathscr{L}^{S}(\Delta x) \boldsymbol{E}^{n}=\boldsymbol{E}^{n}-\Delta x^{n} S\left(Q_{j}^{n}\right)+\frac{\left(\Delta x^{n}\right)^{2}}{2}\left(\frac{\partial S}{\partial E}\right)_{j}^{n} S\left(Q_{j}^{n}\right)
$$

The finite-volume formulation of operator $\mathscr{L}^{F}$ is then described in Section 3.

\section{Streamline Godunov method}

In this section, we describe a Godunov-type scheme for solving Eq. (1) using a second-order ENO interpolation with streamline control volumes for the steady Euler equations in equilibrium flows. 


\subsection{Finite-volume formulation}

Let the marching direction be in the $x$-coordinate and the mesh be oriented such that $x_{j}^{n}=$ constant $=x^{n}$ for all $j$. Here, $n$ and $j$ refer to the marching step number and cell number, respectively. The marching distance, $\Delta x^{n}=x^{n+1}-x^{n}$, is chosen to satisfy the usual Courant-Friedrichs-Lewy (CFL) linear stability condition based on the elementary wave systems of the steady Riemann problem at the cell interfaces of all streamline cells. The computational domain in $x-y$ plane is divided into a system of control volumes or cells which in the $y$-direction are centered at $y_{j}^{n}$ and have a height of $\Delta y_{j}^{n}=y_{j+1 / 2}^{n}-y_{j-1 / 2}^{n}$. The computational domain is shown in Fig. 1.

Here, we apply the splitting technique, i.e. let $S=0$ in the steps $\mathscr{L}^{F}(\Delta x)$ for solving the corresponding Riemann problem of Eq. (1): $\partial \boldsymbol{E} / \partial x+\partial \boldsymbol{F} / \partial y=\mathbf{0}$. Then, the difference equations for the $j$ th zone are obtained by integrating $\partial \boldsymbol{E} / \partial x+\partial \boldsymbol{F} / \partial y=\mathbf{0}$ over the $j$ th cell and applying the divergence theorem. That is

$$
\iint_{D}\left(\frac{\partial \boldsymbol{E}}{\partial x}+\frac{\partial \boldsymbol{F}}{\partial y}\right) \mathrm{d} x \mathrm{~d} y=\int_{\partial D}(\boldsymbol{E}, \boldsymbol{F}) \cdot \overrightarrow{\boldsymbol{n}} \mathrm{d} \ell
$$

where $D$ is the control area or cell, $\partial D$ is the boundary curve of the control area, $\vec{n}$ is the outward unit normal to $\partial D$, and $\mathrm{d} \ell=\sqrt{\mathrm{d} x^{2}+\mathrm{d}^{2}}$ is the infinitesimal arc length on $\partial D$. Expanding Eq. (10), it can be expressed in terms of numerical fluxes as

$$
\boldsymbol{E}_{i}^{n+1}=\boldsymbol{E}_{j}^{n} \frac{\Delta y_{j}^{n}}{\Delta y_{j}^{n+1}}-\frac{\Delta x^{n}}{\Delta y_{j}^{n+1}}\left(\boldsymbol{G}_{j+1 / 2}^{N}-\boldsymbol{G}_{j-1 / 2}^{N}\right),
$$

where the numerical flux $\boldsymbol{G}_{j+1 / 2}^{N}$ is defined as

$$
\boldsymbol{G}_{j+1 / 2}^{N}=\boldsymbol{F}_{j+1 / 2}^{n+1 / 2}-s_{j+1 / 2}^{n} \boldsymbol{E}_{j+1 / 2}^{n+1 / 2}
$$

Here, $s_{j+1 / 2}^{n}$ is the slope of the cell interface at $y_{j+1 / 2}^{n+1 / 2}$, and is given approximately by

$$
s_{j+1 / 2}^{n}=\frac{y_{j+1 / 2}^{n+1}-y_{j+1 / 2}^{n}}{\Delta x^{n}} .
$$

For any quantity $f$, the cell average of $f$ is

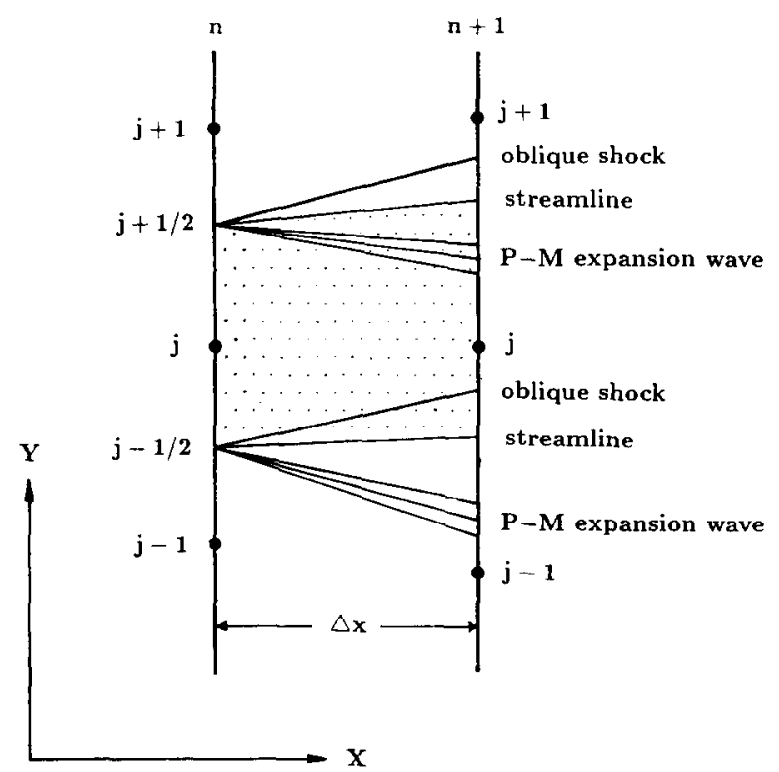

Fig. 1. Strcamline meshes in $(x, y)$ coordinates for the streamline Godunov method. 


$$
f_{j}^{n}=\frac{1}{\Delta y_{j}^{n}} \int_{y_{j-1 / 2}^{n}}^{y_{j+1 / 2}^{n}} f\left(x^{n}, y\right) \mathrm{d} y,
$$

and the distance average of $f$ along the upper and lower boundaries of cells are

$$
f_{j+1 / 2}^{n+1 / 2}=\frac{1}{\Delta x^{n}} \int_{x^{n}}^{x^{n+1}} f\left(x, y_{j+1 / 2}^{n}+s_{j+1 / 2}^{n} \cdot\left(x-x^{n}\right)\right) \mathrm{d} x .
$$

In the first-order Godunov scheme, the $j$ th cell average of state variables $Q_{i}^{n}$, at marching step $n$ is considered as constant within that cell and the numerical fluxes $\boldsymbol{G}_{j+1 / 2}^{N}$ along the interface between the $j$ th cell and the $(j+1)$ th cell from marching step $n$ to $n+1$ is to be obtained from the self-similar solution $\mathrm{R}\left[\left(y-y_{j+1 / 2}^{n}\right) /(x-\right.$ $\left.x^{n}\right) ; Q_{j}^{n}, Q_{j+1}^{n}$ ] at $y=y_{j+1 / 2}^{n+1 / 2}$ to the steady Riemann problem formed by two adjacent piecewise constant flow states $Q_{j}^{n}$ and $Q_{j+1}^{n}$, i.e. with $Q_{j}^{n}=Q_{\mathrm{B}}$ and $Q_{j+1}^{n}=Q_{\mathrm{T}}$ and use the procedure described in Section 4 .

\subsection{Numerical procedure}

The algorithm for computing the interface flux from the initial data, say step $n$, and updating solutions to step $n+1$ may be divided into a number of steps. These are

Step 1. 'Decode' $\boldsymbol{E}_{j}^{n}$ to obtain $\boldsymbol{Q}_{j}^{n}$. Given $\boldsymbol{E}_{j}^{n}$, one has

$$
v=\frac{\mathrm{E}_{3}}{\mathrm{E}_{1}}, \quad H=\frac{\mathrm{E}_{4}}{\mathrm{E}_{1}},
$$

and assuming that $\rho$ is known, then

$$
\begin{aligned}
& u=\frac{\mathrm{E}_{1}}{\rho}, \\
& P=\mathrm{E}_{2}-\frac{\mathrm{E}_{1}^{2}}{\rho} \\
& h=\frac{\mathrm{E}_{4}}{\mathrm{E}_{1}}-\frac{1}{2}\left[\left(\frac{\mathrm{E}_{1}}{\rho}\right)^{2}+\left(\frac{\mathrm{E}_{3}}{\mathrm{E}_{1}}\right)^{2}\right] .
\end{aligned}
$$

From Eq. (18), we define an object function

$$
f(\rho)=P(\rho, h)-\left(\mathrm{E}_{2}-\frac{\mathrm{E}_{1}^{2}}{\rho}\right) .
$$

In Eq. (20), $P(\rho, h)$ comes from equation of state, Eq. (3), such that $h$ is calculated from Eq. (19). Using a numerical iteration procedure, one gets a converged value of $\rho$, after 3 to 5 iterations with the tolerance below $10^{-6}$. Once $\rho$ is obtained, $u$ and $P$ can be determined from Eqs. (17) and (18).

Step 2. Apply second-order ENO representation to evaluate the state variable pair $\left(\overline{\boldsymbol{Q}}_{j+1 / 2}^{+}, \overline{\boldsymbol{Q}}_{j+1 / 2}^{-}\right)$in the upper and lower sides of cell interface. In Eq. (12), the intermediate values $\boldsymbol{E}_{j+1 / 2}^{n+1 / 2}, \boldsymbol{F}_{j+1 / 2}^{n+1 / 2}$ and $\boldsymbol{G}_{j+1 / 2}^{N}$ can be obtained more accurately at the cell interfaces using a second-order ENO interpolation and are given by

$$
\begin{aligned}
& \boldsymbol{E}_{j+1 / 2}^{n-}=\boldsymbol{E}_{j}^{n}+\frac{1}{2} Z_{j}^{n} \Delta y_{j}^{n}, \\
& \boldsymbol{E}_{j-1 / 2}^{n+}=\boldsymbol{E}_{j}^{n}-\frac{1}{2} Z_{j}^{n} \Delta y_{j}^{n},
\end{aligned}
$$

with

$$
Z_{j}^{n}=\mathrm{m}\left(a_{j}, b_{j}\right),
$$

where 


$$
\begin{aligned}
& a_{j}=\frac{\Delta_{+} E_{j}^{n}}{\Delta_{+} y_{j}^{n}}-\eta \Delta y_{j}^{n} \overline{\mathrm{m}}\left(\frac{\Delta_{+} \Delta_{+} E_{j}^{n}}{\Delta_{+} \Delta_{+} y_{j}^{n}}, \frac{\Delta_{-} \Delta_{+} E_{j}^{n}}{\Delta_{-} \Delta_{+} y_{j}^{n}}\right), \\
& b_{j}=\frac{\Delta_{E_{j}} \boldsymbol{E}_{j}^{n}}{\Delta_{-} y_{j}^{n}}-\eta \Delta y_{j}^{n} \overline{\mathrm{m}}\left(\frac{\Delta_{+} \Delta \boldsymbol{E}_{j}^{n}}{\Delta_{+} \Delta_{-} y_{j}^{n}}, \frac{\Delta \boldsymbol{\Delta}_{j}^{n}}{\Delta_{-} \Delta_{-} y_{j}^{n}}\right) .
\end{aligned}
$$

In the above, $\Delta_{ \pm} \boldsymbol{E}_{j}^{n}= \pm\left(\boldsymbol{E}_{j \pm 1}^{n}-\boldsymbol{E}_{j}^{n}\right)$ and $\Delta_{ \pm} y_{j}^{n}= \pm\left(y_{j \pm 1}^{n}-y_{j}^{n}\right)$ denote the usual forward and backward difference operators. In Eq. (23), if $\eta=0$, one has a second-order TVD scheme, and if $\eta=1 / 2$, one has a uniformly second-order ENO scheme. The limiter functions $m$ and $\bar{m}$ are defined, respectively, by

$$
\mathrm{m}(a, b)= \begin{cases}c \min (|a|,|b|), & \text { if } \operatorname{sgn}(a)=\operatorname{sgn}(b)=c, \\ 0, & \text { otherwise }\end{cases}
$$

and

$$
\overline{\mathrm{m}}(a, b)= \begin{cases}a, & \text { if }|a| \leqslant|b|, \\ b, & \text { if }|a|>|b| .\end{cases}
$$

Next, we advance $\boldsymbol{E}_{j \pm 1 / 2}^{n \mp}$ to step $n+1 / 2$ as follows:

$$
\begin{aligned}
& \overline{\boldsymbol{E}}_{j+1 / 2}^{-}=\boldsymbol{E}_{j+1 / 2}^{n-}-\frac{\Delta x^{n}}{2 \Delta y_{j}^{n}}\left[\boldsymbol{F}\left(\boldsymbol{E}_{j+1 / 2}^{n-}\right)-\boldsymbol{F}\left(\boldsymbol{E}_{j-1 / 2}^{n+}\right)\right], \\
& \overline{\boldsymbol{E}}_{j+1 / 2}^{+}=\boldsymbol{E}_{j-1 / 2}^{n+}-\frac{\Delta x^{n}}{2 \Delta y_{j}^{n}}\left[\boldsymbol{F}\left(\boldsymbol{E}_{j+1 / 2}^{n-}\right)-\boldsymbol{F}\left(\boldsymbol{E}_{j-1 / 2}^{n+}\right)\right] .
\end{aligned}
$$

After obtaining $\overline{\boldsymbol{E}}_{j \pm 1 / 2}^{\mp}$, we then calculate $\overline{\boldsymbol{Q}}_{j \pm 1 / 2}^{\mp}$ by using the decoding formula as shown in Step 1 .

Step 3. Obtain the self-similar solutions $\mathrm{R}\left[\left(y-y_{j+1 / 2}^{n}\right) /\left(x-x_{j+1 / 2}^{n}\right) ; \bar{Q}_{j+1 / 2}^{+}, \bar{Q}_{j+1 / 2}^{-}\right]$by solving the steady Riemann problem for equilibrium gases. The solution states along the upper and lower boundaries of cells, $\boldsymbol{Q}_{j \pm 1 / 2}^{n+1 / 2}$, are used to evaluate the numerical fluxes $\boldsymbol{G}_{j \pm 1 / 2}^{N}$ along $\left(y-y_{j \pm 1 / 2}^{n}\right) /\left(x-x_{j \pm 1 / 2}^{n}\right)=s_{j \pm 1 / 2}^{n}$.

Step 4. Generate the streamline meshes. The streamline meshes are carried out by defining

$$
\begin{aligned}
& x_{j}^{n+1}=x_{j}^{n}+\Delta x^{n}, \\
& y_{j \pm 1 / 2}^{n+1}=y_{j \pm 1 / 2}^{n}+\Delta x^{n} s_{j \pm 1 / 2}^{0} .
\end{aligned}
$$

Here, $\Delta x^{n}$ is the marching distance from step $n$ to step $n+1$ and $s_{j \pm 1 / 2}^{0}$ are the slope of the slipline which comes from the Riemann solution at the corresponding cell interfaces, i.e. $s_{j \pm 1 / 2}^{0}=\left(\tan \theta^{*}\right)_{j \pm 1 / 2}^{n+1 / 2}$. From the $\mathrm{CHL}$ criterion, $\Delta x^{n}$ can be estimated by

$$
\Delta x^{n}=\min \left[\frac{\Delta y_{j}^{n}}{\left|s_{j-1 / 2}^{+}\right|+\left|s_{j+1 / 2}^{-}\right|}\right],
$$

where $s_{j \pm 1 / 2}^{\mp}$ are the maximum slope of the,-+ waves at the cell interfaces $y=y_{j \pm 1 / 2}^{n+1 / 2}$, determined from the Ricmann solution at the corresponding cell interfaces. Then, we can determine the ccll height and the $y$-coordinate of the cell at the marching step $n$ as

$$
\Delta y_{j}^{n+1}=y_{j+1 / 2}^{n+1}-y_{j-1 / 2}^{n+1},
$$

and

$$
y_{j}^{n+1}=\frac{1}{2}\left(y_{j+1 / 2}^{n+1}+y_{j-1 / 2}^{n+1}\right)=y_{j}^{n}+\Delta x^{n} \frac{s_{j+1 / 2}^{0}+s_{j-1 / 2}^{0}}{2} .
$$

Note that the mean slope of streamline for the cell is approximately equal to the average of the slopes at the upper and lower interfaces of the cell. 
Step 5. Lastly, the solutions of $x$-direction convection fluxes at step $n+1 \boldsymbol{E}_{j}^{n+1}$, can be obtained from Eq. (11), and then calculate the state variables $Q_{j}^{n+1}$ by using the decoding formula as stated in Step 1 .

At this stage the numerical procedure of the streamline Godunov method for marching solution from step $n$ to step $n+1$ is completed. To march forward further in $x$, one goes back to Step 1 and repeats Steps 1-5.

We note that the procedure to generate the streamline grids described here is slightly different to that described in [1]. In [1], the slope of the cell interface is approximated by the average speed of the upper and lower flow velocities of the interface while here we employ the slope of the slipline (interface) which is from the exact Riemann solution. In most cases, the two approaches give the same results, however, there are cases, particularly with solid boundary, the method in [1] may allow the flow to cut into the solid boundary. Although one can overcome this problem by cutting the size of marching step $\Delta x$, but at high speeds this sometimes can be extremely costly. The present approach improves this and indicates its robustness in practical computations.

If a solid boundary is present in the flow, it must be a streamline. At this wall the local velocity vector must be tangent to the solid wall. So the boundary condition to be imposed on the solid boundary is

$$
\frac{v_{w}}{u_{w}}=\tan \theta_{w},
$$

where $\theta_{w}$ is the inclination of the solid wall and $u_{w}$ and $v_{w}$ are the corresponding components of the local flow velocity parallel to the surface of the solid wall. This results in a boundary Riemann problem for equilibrium gases and is solved using the procedure described in Section 4.

\section{Riemann problem for 2-D steady supersonic flows of equilibrium gases}

The Riemann problem for two-dimensional steady supersonic flows for equilibrium gases is specified as the system (1) of hyperbolic conservation laws subject to the initial condition

$$
Q\left(x=x_{0}, y\right)= \begin{cases}Q_{\mathrm{T}}, & y>y_{0} \\ Q_{\mathrm{B}}, & y \leqslant y_{0}\end{cases}
$$

where $Q=(\rho, u, v, P)^{\top}$ and subject to the equation of state

$$
P=P(\rho, e),
$$

where the flow states are given at the initial data line $x=x_{0}$ and $Q_{\mathrm{T}}$ and $Q_{\mathrm{B}}$ denote the top and bottom states. The equation of state is prescribed as a given function of any two thermodynamic variables, such as $\rho, e$.

The solution to the above steady Riemann problem for equilibrium gases is self-similar in the variable $\left(y-y_{0}\right) /\left(x-x_{0}\right)$ and consists of three types of elementary wave $+, 0,-$, namely, the oblique shock waves, the slip lines, and the Prandtl-Meyer expansion waves, in that order from top to bottom, as shown in Fig. 2. The \pm -waves may be either shocks or expansions while 0 -wave is always a slipline. In order to construct these waves, one needs to determine the intersection $\left(P^{*}, \theta^{*}\right)$ in the $P-\theta$ plane, as shown in Fig. 3. It is noted that although the classification of the elementary waves for equilibrium gases is the same as that for perfect gases, nevertheless, the real gas effects complicate the formula considerably and exact formula cannot be found explicitly and numcrical solution has to be pursucd. Denotc this Riemann solution as $\mathrm{R}\left[\left(y-y_{0}\right) /(x-\right.$ $\left.\left.x_{0}\right) ; Q_{\mathrm{T}}, Q_{\mathrm{B}}\right]$. These elementary waves can be used to construct a solution to the steady Riemann problem for two-dimensional supersonic flows.

\subsection{Elementary wave systems for equilibrium gases}

Let $Q_{0}$ and $Q$ be states ahead of and behind any one of the elementary waves, then there are three cases:

(A) The wave is a slipline $\left(P=P_{0}\right)$

In this case, from the physical feature of the streamline, we have 

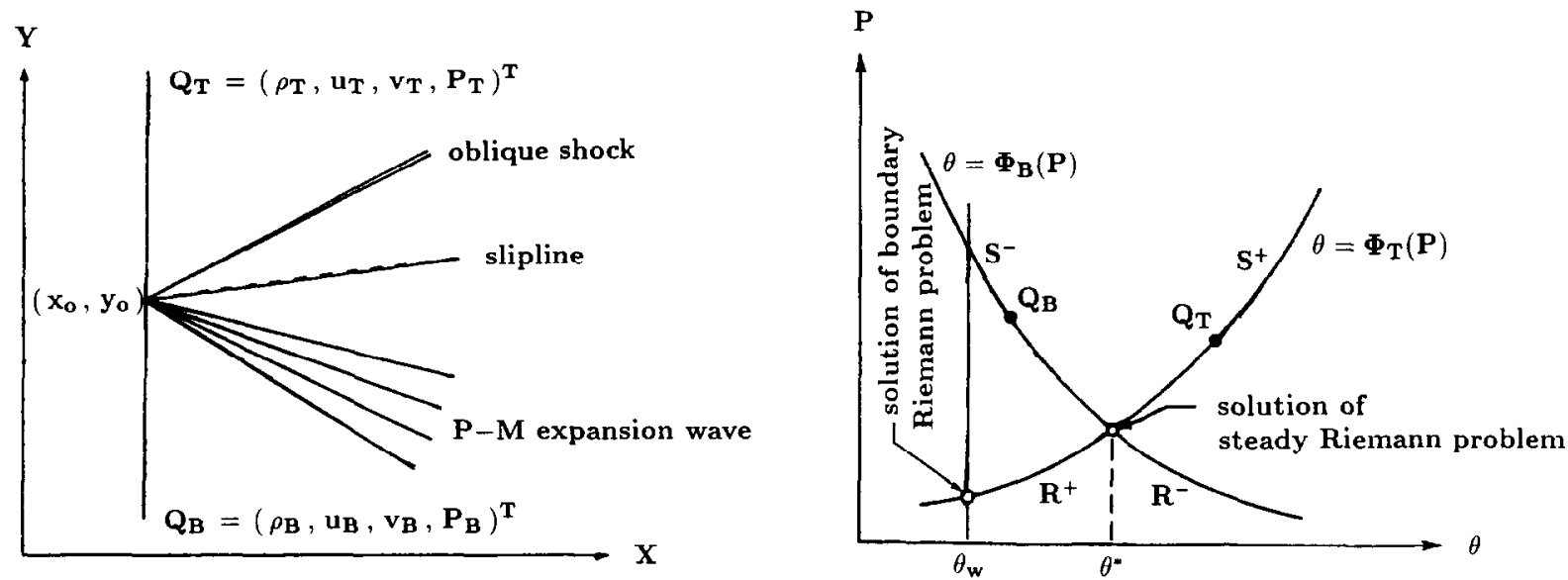

Fig. 2. Elementary wave system in the solution of steady Riemann Problem.

Fig. 3. Solution for the steady Riemann problem in the $(P, \theta)$ plane.

$$
\begin{aligned}
& P(\rho, e)=P_{0}\left(\rho_{0}, e_{0}\right)=P^{*}, \\
& \theta=\tan ^{-1}\left(\frac{v}{u}\right)=\theta_{0}=\tan ^{-1}\left(\frac{v_{0}}{u_{0}}\right)=\theta^{*} .
\end{aligned}
$$

That is, the pressure $P$ and flow angle $\theta$ must be identical on either side of the slipline, which holds for both perfect and equilibrium gases. The density $\rho$, velocity components and the other thermodynamic variables may jump abruptly.

(B) The wave is an oblique shock wave $\left(P>P_{0}\right)$

In this case, from the oblique shock relations and the equation of state, we have

$$
\begin{gathered}
\rho_{0} V_{0}^{2} \sin ^{2} \beta=\frac{P-P_{0}}{1-\rho_{0} / \rho}, \\
\tan \delta=\frac{\left(\rho-\rho_{0}\right) \tan \beta}{\rho+\rho_{0} \tan ^{2} \beta},
\end{gathered}
$$

where $V_{0}=\sqrt{u_{0}^{2}+v_{0}^{2}}, \beta$ is the shock angle, and $\delta=\Delta \theta$ is the flow deflection angle.

The changes of the thermodynamic variables across an oblique shock wave can be expressed by the Rankine-Hugoniot relation,

$$
e-e_{0}=\frac{P+P_{0}}{2}\left(\frac{1}{\rho_{0}}-\frac{1}{\rho}\right),
$$

or

$$
\frac{\rho_{0}}{\rho}=1-2 \rho_{0} \frac{e(P, \rho)-e_{0}}{P+P_{0}},
$$

This Rankine-Hugoniot jump condition is an implicit relation between pressure $P$ and density $\rho$. If $P$ is known, $\rho$ can be obtained by letting the object function be

$$
\tilde{f}(\rho)=\frac{\rho_{0}}{\rho}+2 \rho_{0} \frac{e(P, \rho)-e_{0}}{P+P_{0}}-1,
$$

from using the appropriate numerical procedure.

Having obtained the flow properties ahead of and behind the oblique shock wave, we can get $\beta$ and $\delta$ from 
Eqs. (36) and (37). The flow angle $\theta$ is then

$$
\begin{cases}\theta=\Phi_{\mathrm{T}}(P)=\theta_{0}+\delta, & \text { (for the flow on the top) } \\ \theta=\Phi_{\mathrm{B}}(P)=\theta_{0}-\delta, & \text { (for the flow on the bottom) }\end{cases}
$$

(C) The wave is a Prandt-Meyer expansion wave $\left(P<P_{0}\right)$

In this case we use the isentropic condition and $H=$ const., then we have

$$
T \mathrm{~d} S=\mathrm{d} h-\frac{1}{\rho} \mathrm{d} P=0,
$$

where $S$ is the specific entropy and

$$
\mathrm{d} H=\mathrm{d} h+V \mathrm{~d} V=0 .
$$

Combining above two relations, we obtain

$$
\frac{\mathrm{d} V}{V}=-\frac{\mathrm{d} P}{\rho V^{2}} .
$$

From the theory of the Prandtl-Meyer flow for equilibrium gases, we have the local flow inclination angle $\mathrm{d} \theta$ as

$$
\mathrm{d} \theta=\mp \sqrt{M^{2}-1} \frac{\mathrm{d} V}{V} .
$$

By using Eq. (44), Eq. (45) can be rewritten as

$$
\mathrm{d} \theta= \pm \frac{\sqrt{M^{2}-1}}{\rho V^{2}} \mathrm{~d} P,
$$

or the flow deflection angle $\delta$

$$
\delta=\theta-\theta_{0}= \pm \int_{P^{0}}^{P} \frac{\sqrt{M^{2}-1}}{\rho V^{2}} \mathrm{~d} P .
$$

The speed of sound and the local flow velocity for an equilibrium gas can be calculated from

$$
a^{2}=\frac{\mathrm{d} P}{\mathrm{~d} \rho} ; \quad V^{2}=2(H-h)=2\left(H-\int \frac{\mathrm{d} p}{\rho}\right),
$$

and then the local Mach number for an equilibrium gas is

$$
M^{2}=\frac{V^{2}}{a^{2}} \text {. }
$$

With $P$ is known, the flow inclination angle $\theta$ after a Prandtl-Meyer expansion wave becomes

$$
\begin{cases}\theta=\Phi_{\mathrm{T}}(P)=\theta_{0}+\int_{P_{0}}^{P} \frac{\sqrt{M^{2}-1}}{\rho V^{2}} \mathrm{~d} P, & \text { (for the flow on the top) } \\ \theta=\Phi_{\mathrm{B}}(P)=\theta_{0}-\int_{P_{0}}^{P} \frac{\sqrt{M^{2}-1}}{\rho V^{2}} \mathrm{~d} P, & \text { (for the flow on the bottom) }\end{cases}
$$

The integrals in Eq. (48) can be evaluated numerically using Simpson's rule. They play the same role in equilibrium flow as the Prandtl-Meyer function does in a perfect gas flow.

Therefore, through any state $Q_{0}$ at $\mathscr{L}^{F}(\Delta x)$-steps, with $P / P_{0}$ as parameter, there are two families of states connecting to $Q_{0}$, namely, the compression state $\left(P / P_{0} \geqslant 1\right)$, and the expansion state $\left(P / P_{0} \leqslant 1\right)$. The two families join smoothly and can be regarded as a single family. For example, in the plane, the two curves form a single smooth curve at $Q=Q_{0}$, and in the $P-\theta$ plane, a constant $P=P^{*}$ line can be drawn and the value of $\theta^{*}$ can be determined. 


\subsection{Solution procedure of Riemann problem}

(i) In the $P-\theta$ plane, there are two curves that pass through the states $Q_{\mathrm{T}}$ and $Q_{\mathrm{B}}$; they are defined respectively by

$$
\theta=\Phi_{\mathrm{T}}(P)= \begin{cases}\theta_{\mathrm{T}}+\tan ^{-1}\left[\frac{\left(\rho-\rho_{\mathrm{T}}\right) \tan \beta}{\rho+\rho_{\mathrm{T}} \tan ^{2} \beta}\right], & P>P_{\mathrm{T}} \\ \theta_{\mathrm{T}}+\int_{P_{\mathrm{T}}}^{P} \frac{\sqrt{M^{2}-1}}{\rho V^{2}} \mathrm{~d} P, & P \leqslant P_{\mathrm{T}}\end{cases}
$$

and

$$
\theta=\Phi_{\mathrm{B}}(P)= \begin{cases}\theta_{\mathrm{B}}-\tan ^{-1}\left[\begin{array}{c}
\left(\rho-\rho_{\mathrm{B}}\right) \tan \beta \\
\rho+\rho_{\mathrm{B}} \tan ^{2} \beta
\end{array}\right], & P>P_{\mathrm{B}} \\
\theta_{\mathrm{B}}-\int_{P_{\mathrm{B}}}^{P} \frac{\sqrt{M^{2}-1}}{\rho V^{2}} \mathrm{~d} P, & P \leqslant P_{\mathrm{B}}\end{cases}
$$

These curves are depicted in Fig. 3.

(ii) Using appropriate numerical procedure to solve $P^{*}$ and $\theta^{*}$. Define the object function as follows:

$$
f(P)=\Phi_{\mathrm{T}}(P)-\Phi_{\mathrm{B}}(P) .
$$

Then, the Riemann solution becomes

$$
f\left(P^{*}\right)=0 ; \quad \theta^{*}=\Phi_{\mathrm{T}}\left(P^{*}\right)=\Phi_{\mathrm{B}}\left(P^{*}\right) .
$$

(iii) With the values $P^{*}$ and $\theta^{*}$ are known, we can calculate all other flow variables using appropriate equations for compression and expansion states on both sides of the slipline. These flow quantities in the two uniform flow regions adjacent to the slipline are all that is needed for computing the interface convection fluxes $\boldsymbol{E}_{j \pm 1 / 2}^{n+1 / 2}, \boldsymbol{F}_{j \pm 1 / 2}^{n+1 / 2}$ and the numerical fluxes $\boldsymbol{G}_{j \pm 1 / 2}^{N}$ in our streamline Godunov method.

At a solid wall the flow tangency condition requires that the solid wallis also a streamline. This causes a boundary Riemann problem and is specified as the system (1) of hyperbolic conservation laws subject to the condition

$$
\begin{cases}Q\left(x=x_{0}, y\right)=Q_{\mathrm{T}}, & y>y_{w}, \\ \theta=\theta^{*}=\theta_{w}, & y=y_{w}\end{cases}
$$

Here, we assume that the flow is on the top of solid wall. In this way the tangency boundary condition is automatically satisfied. The solution procedure is then the same as that described above. That is, since

$$
\theta_{w}=\Phi_{\mathrm{T}}(P)= \begin{cases}\theta_{\mathrm{T}}+\tan ^{-1}\left[\frac{\left(\rho-\rho_{\mathrm{T}}\right) \tan \beta}{\rho+\rho_{\mathrm{T}} \tan ^{2} \beta}\right], & \theta_{w}>\theta_{\mathrm{T}} \\ \theta_{\mathrm{T}}+\int_{P_{\mathrm{T}}}^{P} \frac{\sqrt{M^{2}-1}}{\rho V^{2}} \mathrm{~d} P, & \theta_{w} \leqslant \theta_{\mathrm{T}}\end{cases}
$$

then the boundary Riemann solution becomes

$$
f_{w}\left(P^{*}\right)=\Phi_{\mathrm{T}}\left(P^{*}\right)-\theta_{w}=0,
$$

for the flow on the top side of solid wall. Similar result can be obtain for the flow on the bottom side of solid wall.

The above elementary solutions of the steady Riemann problem for equilibrium gases are used in our streamline Godunov method described in previous section. Also, the Riemann solver discussed here can be used to obtain the exact solution for some of the test problems in both perfect gas and equilibrium flows. 


\section{Specification of real gas models}

In this section we describe the two equilibrium gas models used to calculate the equilibrium gas properties.

\subsection{Tannehill's equilibrium air gas table}

To compute the thermodynamic properties of equilibrium air, we must first determine the species concentrations at the given $T$ and $P$ of the mixture. Some typical chemical reactions in equilibrium condition for air are

$$
\begin{aligned}
& \mathrm{O}_{2} \rightleftharpoons \mathrm{O}+\mathrm{O}, \\
& \mathrm{N}_{2} \rightleftharpoons \mathrm{N}+\mathrm{N}, \\
& \mathrm{N}+\mathrm{O} \rightleftharpoons \mathrm{NO}, \\
& \mathrm{N}+\mathrm{O} \rightleftharpoons \mathrm{NO}^{+}+\mathrm{e}^{-} .
\end{aligned}
$$

Generally, there are a total of 22 chemical reactions that occur for temperatures up to about $15000^{\circ} \mathrm{K}$, resulting in a set of 28 nonlinear equations. Each of these reactions will have an equilibrium constant $K_{P}(T)$. These equilibrium constants are known functions of temperature from cither measurements or statistical mechanics computations. These equilibrium constants are related to the partial pressures of the individual constituents by the law of mass action, such that

$$
\begin{aligned}
& \frac{\left(P_{\mathrm{O}}\right)^{2}}{P_{\mathrm{O}_{2}}}=K_{P, \mathrm{O}_{2}}(T), \\
& \frac{\left(P_{\mathrm{N}}\right)^{2}}{P_{\mathrm{N}_{2}}}=K_{P, \mathrm{~N}_{2}}(T), \\
& \frac{P_{\mathrm{NO}}}{P_{\mathrm{N}} P_{\mathrm{O}}}=K_{P, \mathrm{NO}}(T), \\
& \frac{P_{\mathrm{NO}^{+}} P_{\mathrm{e}^{-}}}{P_{\mathrm{N}} P_{\mathrm{O}}}=K_{P, \mathrm{NO}^{+}}(T) .
\end{aligned}
$$

There would be a single equation corresponding to each significant chemical reaction. For temperatures below about $9000^{\circ} \mathrm{K},(56)-(59)$ are the most important chemical reactions. Mathematically, for the constituents of chemical reactions (56)-(59), Dalton's law of partial pressure can be written as follows:

$$
P=P_{\mathrm{O}_{2}}+P_{\mathrm{O}}+P_{\mathrm{N}_{2}}+P_{\mathrm{N}}+P_{\mathrm{NO}}+P_{\mathrm{NO}^{+}}+P_{\mathrm{e}^{-}} \text {. }
$$

Of course, if the temperature is higher than $9000{ }^{\circ} \mathrm{K}$ and other chemical reactions occur, and other species must be added to Eq. (64), just as the chemical reaction equations are included in the set (56) through (59). Note that there are seven unknowns in Eq. (64) and we have only five equations so far. The remaining two equations come from a chemical balance of the number of $O$ and $N$ atoms and the fact that electric charge must be conserved. These facts, in equation form, may be written as

$$
\frac{2 P_{\mathrm{O}_{2}}+P_{\mathrm{O}}+P_{\mathrm{NO}}+P_{\mathrm{NO}^{+}}}{2 P_{\mathrm{N}_{2}}+P_{\mathrm{N}}+P_{\mathrm{NO}}+P_{\mathrm{NO}^{+}}} \approx \frac{0.21}{0.79},
$$

and

$$
P_{\mathrm{NO}^{+}}=P_{\mathrm{e}^{-}} .
$$

For given temperature and pressure, Eqs. (60)-(66) give a unique set of seven partial pressures for the seven species. This is a nonlinear set of algebraic equations that must be solved for each given $T$ and $P$ to determine the species content once temperatures are high enough for dissociation to occur. Knowing the partial pressures of the species present allows one to uniquely determine the other properties of the system.

If we focus on how to compute the thermodynamic and flowficld propertics of a chemically equilibrium 
reacting gas, the properties of air as a function of $P$ and $\rho$ could be used in a table look-up mode in the computation process. A more simplified approach was produced by Srinivasan et al. [9]. They produced curve fits from algebraic equations for $P=P(e, \rho), a=a(e, \rho), T=T(e, \rho), h=h(P, \rho)$ and $T=T(P, \rho)$. These curve fits are valid up to temperatures of $25000^{\circ} \mathrm{K}$. Since this method is very computationally efficient so we adopt this technique in our computation. The details will not be repeated here and can be found in [9].

\subsection{Lighthill's ideal dissociating gas model}

Consider a simple dissociation-recombination reaction for a symmetrical (or homonuclear) diatomic gas $A_{2}$ in equilibrium condition:

$$
A_{2} \rightleftharpoons A+A \text {. }
$$

Introduce a new variable $\alpha$ called the mass fraction of atomic species $A$ of dissociated gas, i.e., $\alpha=\rho_{A} / \rho$, and $\alpha=0 \sim 1$ such that $\alpha=0$ denotes a completely combined gas and $\alpha=1$ a completely dissociated gas. We also have $\rho_{A_{2}} / \rho-1-\alpha$. The equilibrium state of the gas is defined by equation of state which is then taken in the form

$$
P=P(\rho, T) .
$$

The thermodynamic changes in a real gas at high temperatures are rather complicated and are not completely understood. In order to simplify the complicated expressions of the symmetric diatomic gas for easy use in gas dynamics, Lighthill proposed a simplified model which is termed the ideal dissociating gas to describe the changes of state of all gases with reasonable accuracy within a specified range of temperatures and pressures [10]. The law of mass action, which determines the equilibrium composition of the mixture of atoms and molecules, is

$$
\frac{\alpha^{2}}{1-\alpha}=\frac{\rho_{d}}{\rho} \mathrm{e}^{-\Theta_{D} / T}
$$

where $\rho_{d}$ is the characteristic density for dissociation which is a complicated function of $T$ and $\Theta_{D}$ is the characteristic temperature for dissociation. Lighthill showed that it is a reasonable approximation to take $\rho_{d}=$ constant over a wide range of temperatures for $\mathrm{O}_{2}$ and $\mathrm{N}_{2}$. This greatly simplifies the problem; it is consistent with taking the vibrational degrees of freedom of the molecules as always being half - excited, even at low temperatures. In other words, the contribution from the various degrees of freedom to the energy is the same for atoms and molecules. The internal energy per unit mass, $e$, is then

$$
e=R_{A_{2}}\left[3 T+\Theta_{D} \alpha\right] \text {, }
$$

where $R_{A_{2}}$ is the gas constant per unit mass for the diatomic species $A_{2}$, and $R_{A_{2}} \Theta_{D} \alpha$ is the chemical potential energy (the dissociation energy) of the reaction (67).

If a partially dissociated gas, in conditions of thermal equilibrium, can be regarded as a mixture of two perfect gases, the molecules $A_{2}$ and the atoms $A$, with the definition of molecular weight of the mixture, $M_{\text {mix }}$, given by

$$
M_{\text {mix }}=\left[\sum_{j=1}^{n_{s}} \frac{\alpha_{j}}{M_{j}}\right]^{1}=\left[\frac{\alpha}{M_{A}}+\frac{1-\alpha}{M_{A_{2}}}\right]^{-1}=\frac{M_{A_{2}}}{1+\alpha},
$$

where $n_{s}$ is the number of species in the mixture (here $n_{s}=2$ ). Then the gas constant of mixture, $R_{\text {mix }}$, becomes

$$
R_{\text {mix }}=\frac{\tilde{R}}{M_{\text {mix }}}=(1+\alpha) \frac{\tilde{R}}{M_{A_{2}}}=(1+\alpha) R_{A_{2}},
$$

where $\tilde{R}$ is the universal gas constant. Using this $R_{\mathrm{mix}}$, the thermal equation of state of the ideal dissociating gas, 
thereby defining Eq. (68), is taken as

$$
P=\rho R_{\mathrm{mix}} T=(1+\alpha) \rho R_{A_{2}} T .
$$

Combining Eqs. (70) and (71), we have the caloric equation of state

$$
h=e+\frac{P}{\rho}=R_{A_{2}}\left[(4+\alpha) T+\Theta_{D} \alpha\right]
$$

It can be seen that, as $\alpha$ approaches zero at low temperature, the ideal dissociating gas becomes a perfect gas with constant specific heats $c_{p 0}, c_{v 0}$ and with the specific heat ratio $\gamma_{0}=c_{p 0} / c_{v 0}=4 / 3$. This incorrect low-temperature behavior sets a low limit below which air cannot be accurately represented by this model of ideal dissociating gas. An upper limit will also be fixed by the fact that electronic contribution to the internal energy is neglected, as is the ionization. Lighthill sets these lower and upper limits at approximately 1000 and $7000^{\circ} \mathrm{K}$ for $\mathrm{O}_{2}$ and $\mathrm{N}_{2}$ with density range from $10^{-3}$ to 1 . Suggested values of $\rho_{d}$ are [16]

$$
\begin{cases}\rho_{d}=1.5 \times 10^{5} \mathrm{Kg} / \mathrm{m}^{3}, & \text { for } \mathrm{O}_{2} \\ \rho_{d}=1.3 \times 10^{5} \mathrm{Kg} / \mathrm{m}^{3}, & \text { for } \mathrm{N}_{2}\end{cases}
$$

Rewriting the specific enthalpy $h$ from Eq. (72) in terms of $\rho$ and $P$, we have the useful relation $h=h(\rho, P)$ of the form

$$
h(\rho, P)=\frac{4+\alpha}{1+\alpha} \frac{P}{\rho}+R_{A_{2}} \Theta_{D} \alpha .
$$

Then, with the specific enthalpy derivatives $h_{\rho}, h_{P}, h_{\alpha}$ and the relation $\alpha=\alpha(\rho, P)$ from the combination of Eqs. (69) and (71), we have the equilibrium speed of sound as

$$
\begin{aligned}
a^{2} & =\frac{-h_{\rho}-h_{\alpha} \alpha_{\rho}}{h_{P}+h_{\alpha} \alpha_{p}-1 / \rho} \\
& =R_{A_{2}} T \frac{\alpha\left(1-\alpha^{2}\right)\left(1+2 T / \Theta_{D}\right)+\left(8+3 \alpha-\alpha^{3}\right)\left(T / \Theta_{D}\right)^{2}}{\alpha(1-\alpha)+3(2-\alpha)\left(T / \Theta_{D}\right)^{2}} .
\end{aligned}
$$

We can also compute the entropy difference for the ideal dissociation gas:

$$
\begin{aligned}
\frac{S-S_{0}}{R_{A_{2}}}= & 3 \log \left(\frac{T}{T_{0}}\right)-\left(1+\alpha_{0}\right) \log \left(\frac{\rho}{\rho_{0}}\right)+\left(\alpha-\alpha_{0}\right) \log \left(\frac{\rho_{d}}{\rho}\right) \\
& +[(1+\alpha)-(1-\alpha) \log (1-\alpha)-2 \alpha \log \alpha] \\
& -\left[\left(1+\alpha_{0}\right)-\left(1-a_{0}\right) \log \left(1-\alpha_{0}\right)-2 \alpha_{0} \log \alpha_{0}\right],
\end{aligned}
$$

where subscript ' 0 ' denotes the reference condition.

\section{Numerical results and discussions}

In this section, we first test the performance of the present second-order essentially non-oscillatory streamline Godunov method by solving several simple initial and boundary value Riemann problems of steady equilibrium flows and comparing the results with exact solutions. Computations of practical supersonic/hypersonic equilibrium flows are also included. All computations were done with a uniform grid at the initial marching step and with $\mathrm{CFL}=1.0$. Also note that the reference conditions used in all numerical computation cases are: $P_{\text {ref }}=101330 \mathrm{~N} / \mathrm{m}^{2}, \rho_{\text {ref }}=1.292 \mathrm{Kg} / \mathrm{m}^{3}$ and $T_{\text {ref }}=273^{\circ} \mathrm{K}$.

To test the new streamline procedure as defined by (28), we consider several simple test problems. Firstly, we 
consider a model test case of initial-value Riemann problem. It is formed by two parallel supersonic streams at $y_{0}-0.5$ with different states. The initial conditions are given by

$$
\begin{cases}\left(\rho_{\mathrm{T}}, P_{\mathrm{T}}, M_{\mathrm{T}}, \theta_{\mathrm{T}}\right)=\left(0.5,0.25,4.0,0.0^{\circ}\right), & y>0.5 \\ \left(\rho_{\mathrm{B}}, P_{\mathrm{B}}, M_{\mathrm{B}}, \theta_{\mathrm{B}}\right)=\left(1.0,1.00,2.4,0.0^{0}\right), & y<0.5\end{cases}
$$

This is a case considered by Glaz and Wardlaw [15] using Eulerian method and also considered by Loh and Hui [17] using Lagrangian method. In the exact solution, the resulting interaction produces an oblique shock wave, a Prandtl-Mcycr expansion, and a slipline in between. Here, we use 100 cells and the initial cell size is $\Delta y_{\text {ini }}=0.01$. Numerical results for the flow properties at section $x=0.8$ are shown in Fig. 4 along with the exact solution which was obtained using the real gas Riemann solver described in Section 4. Results obtained using both first-order Godunov scheme and ENO2 scheme are shown. The ENO2 results indicate better resolution at

(a)

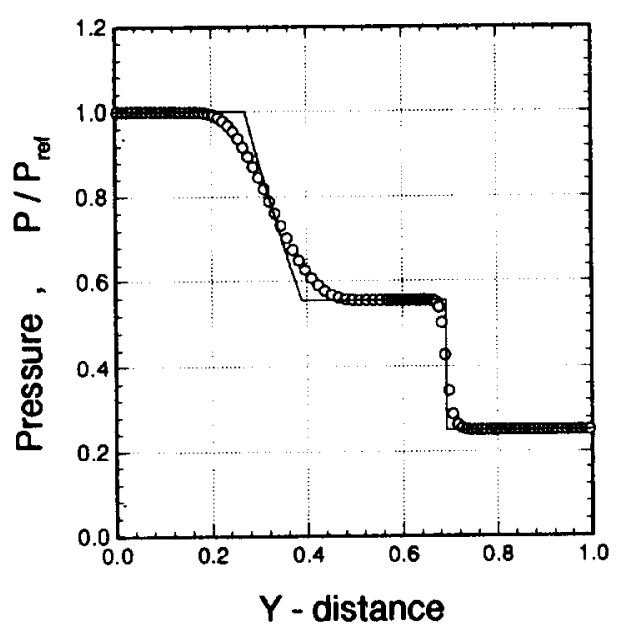

(c)

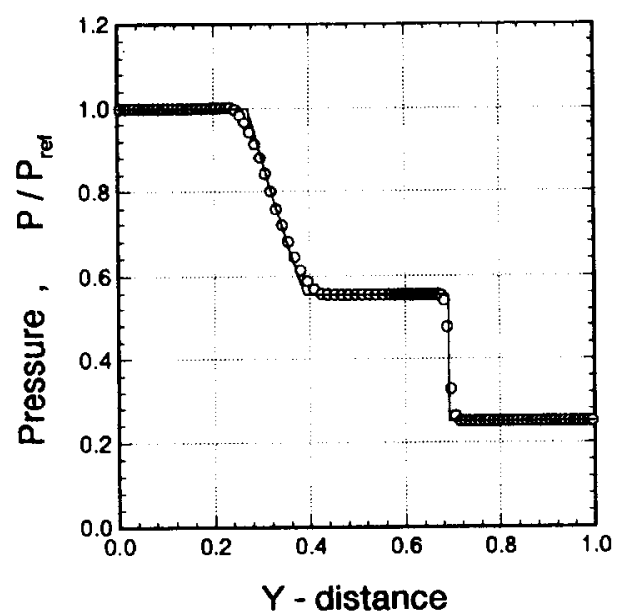

(b)

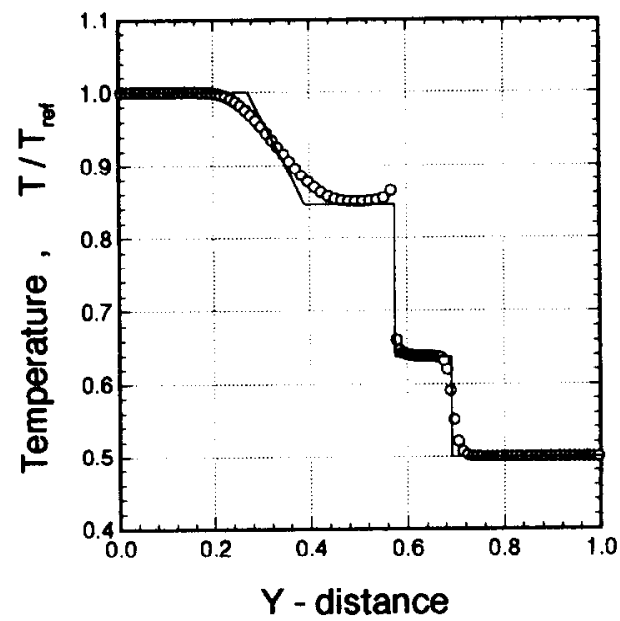

(d)

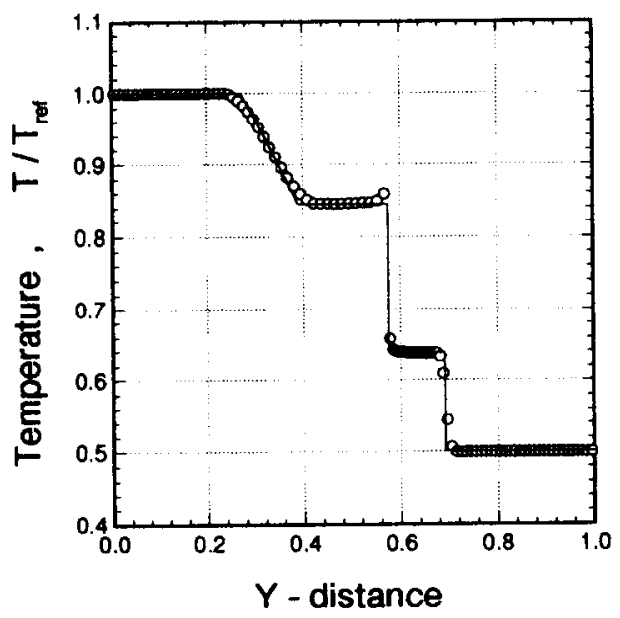

Fig. 4. Solution of initial-value Riemann problem for a confluence of two parallel supersonic stream. A comparison of numerical results (circles) and exact solution (solid line). (a) and (b) first-order Godunov scheme; (c) and (d) second-order FNO scheme. 
shocks and Prandtl-Meyer expansions. By using the streamline mesh system, a better resolution of slipline, even for the first-order scheme, can be achieved. To test the robustness of this method, a more severe case with $M_{\mathrm{T}}=12$ is considered. The results are shown in Fig. 5 at section $x=0.8$. It is observed that the quality of the resolution for shock and slipline remain unchanged even with increasing Mach number. This is another feature of using streamline meshes as computational cells.

The second test case is the same initial-value Riemann problem but formed by a confluence of two intersecting supersonic streams at $y_{0}=0.5$ with different states. The initial conditions are given by

$$
\begin{cases}\left(\rho_{\mathrm{T}}, P_{\mathrm{T}}, M_{\mathrm{T}}, \theta_{\mathrm{T}}\right)=\left(0.2,0.1,4.0,0.0^{0}\right), & y>0.5 \\ \left(\rho_{\mathrm{B}}, P_{\mathrm{B}}, M_{\mathrm{B}}, \theta_{\mathrm{B}}\right)=\left(1.0,1.00,6.0,15.0^{0}\right), & y<0.5\end{cases}
$$

This is clearly a more severe case than the above test case of Riemann problem, but the resulting interaction

(a)

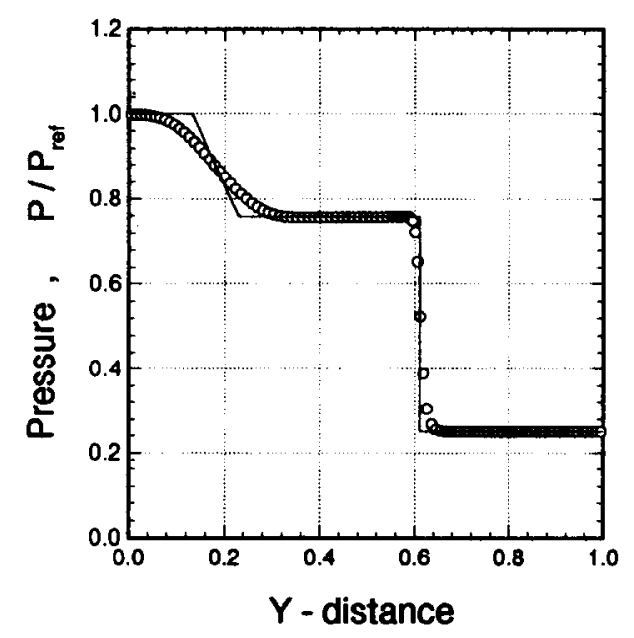

(c)

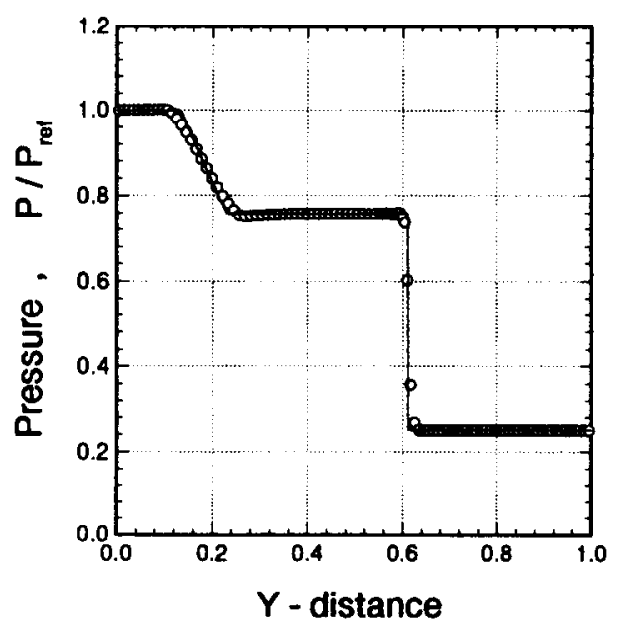

(b)

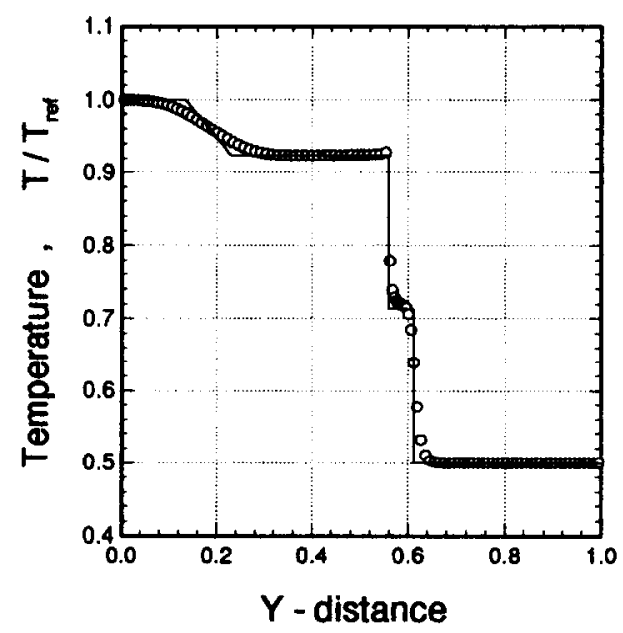

(d)

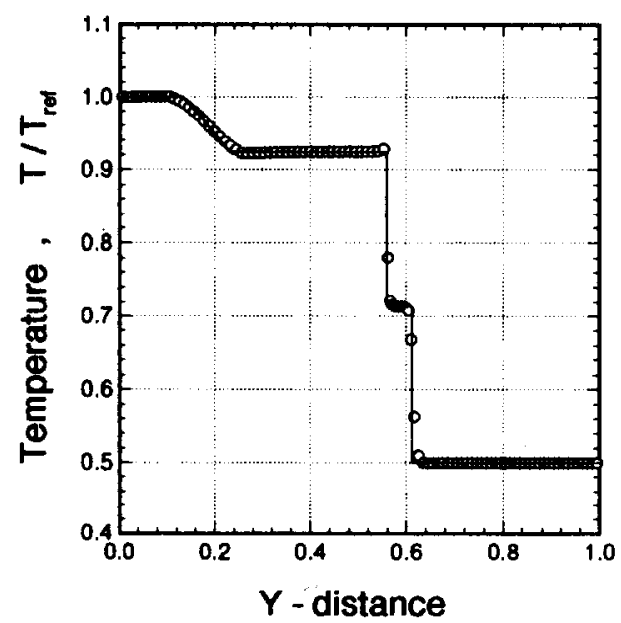

Fig. 5. Solution of initial-value Riemann problem with high Mach number $M_{\mathrm{T}}=12$. A comparison of numerical results (circles) and exact solution (solid line). (a) and (b) first-order Godunov scheme; (c) and (d) second-order ENO scheme. 
produces similar features, namely an oblique shock adjacent to the low-pressure stream side and a PrandtlMeyer expansion flow on the high-pressure stream side. Here we also use 200 cells and $\Delta y_{\mathrm{ini}}=0.005$. Numerical results for the flow properties at section $x=0.8$ are shown in Fig. 6 along with the exact solution. Again, it is seen that shock and slipline discontinuities are sharply resolved by our Godunov-type scheme based on the streamline meshes. In the same manner, the ENO2 results give a better resolution at shock, slipline discontinuity, and also expansion fan structure. This may be attributed to the streamline meshes, shown in Fig. 7 (a) and (b), which follow the movement of the fluid particles. The clustering of the grids at high gradient region such as shock wave can also be observed.

As an cxamplc of initial and boundary value Riemann problems we compute a supersonic flow with $M_{\propto}-5$

(a)

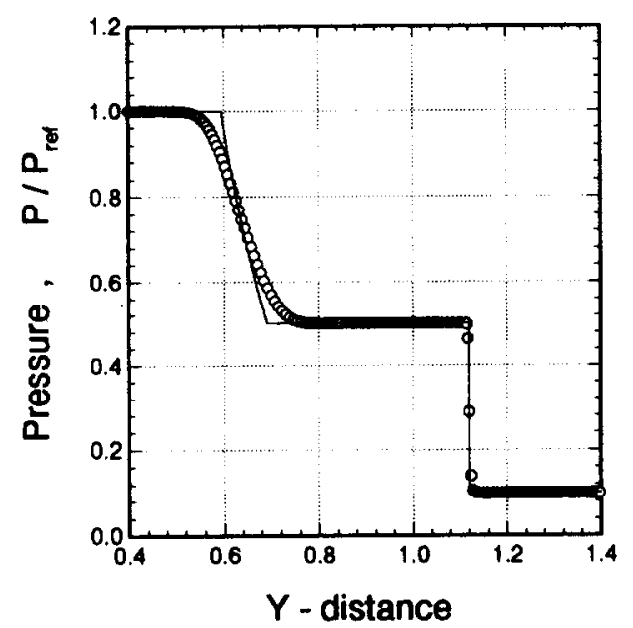

(c)

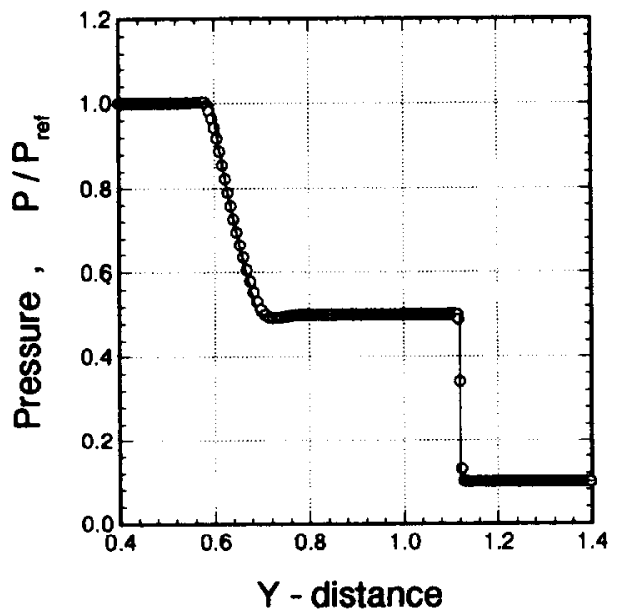

(b)

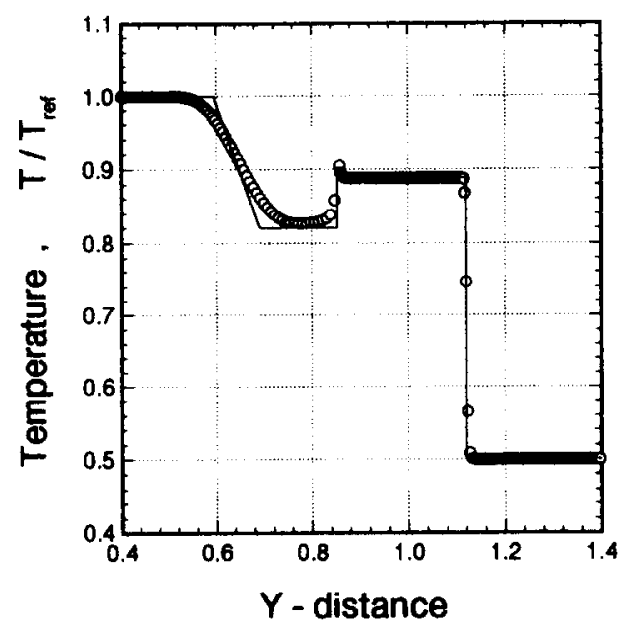

(d)

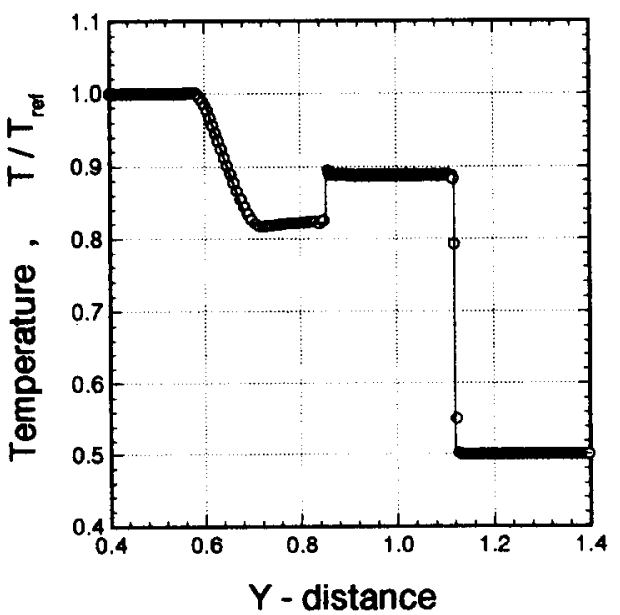

Fig. 6. Solution of initial-value Riemann problem for a confluence of two intersecting supersonic streams. A comparison of numerical results (circles) and exact solution (solid line). (a) and (b) first-order Godunov scheme; (c) and (d) second-order ENO scheme. 
(a)

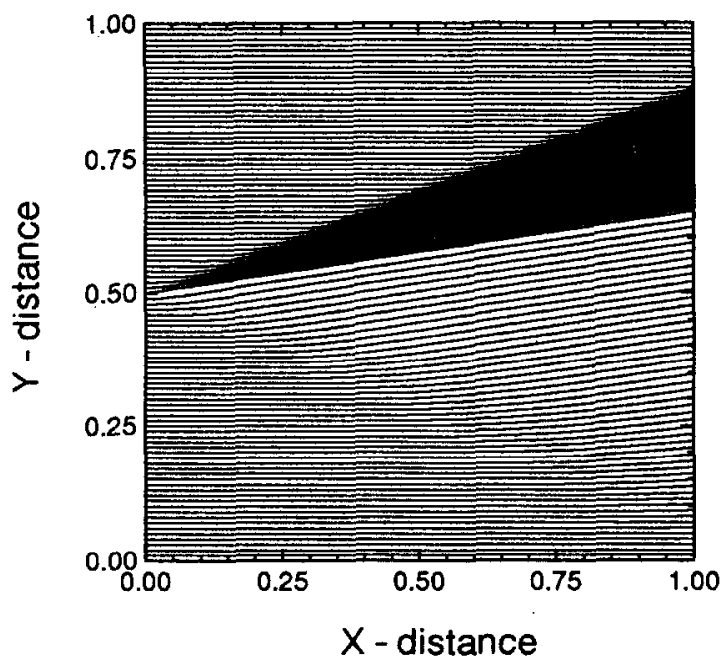

(b)

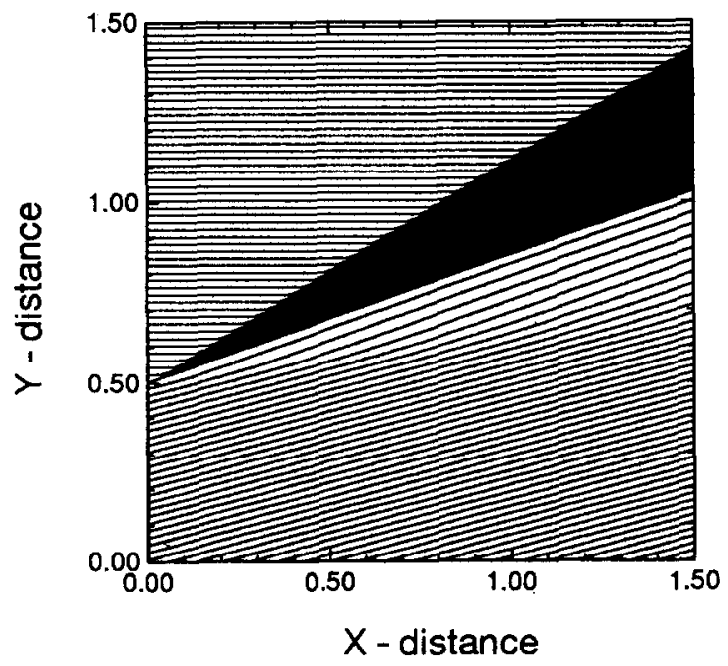

Fig. 7. Streamline grids of initial-value Riemann problem. (a) A confluence of two parallel supersonic streams; (b) a confluence of two intersecting supersonic streams.

past a $20^{\circ}$ wedge. We use 70 cells and $\Delta y_{1 n 1}=0.01$. Numerical results for the flow properties at section $x=1.0$ are plotted in Fig. 8 and compared to the exact solution which was obtained using the boundary Riemann solver. A good accuracy for the flow properties within the shock layer and a better resolution at shock can be seen.

We also compute the Prandtl-Meyer flow with $M_{x}=5$ past a $10^{\circ}$ turning angle. Here 25, 50, 250, cells are employed with $\Delta y_{\mathrm{ini}}=0.01,0.005,0.001$, respectively. Numerical results for the flow properties at section $x=0.5$ are plotted in Fig. 9 and compared with the exact solution which was also obtained using the boundary Riemann solver. Due to the intrinsic feature of streamline, grid size should be widened after expansion and the accuracy of computation would deteriorate, especially at the tail of expansion fan. To attain the same level of accuracy in representation of expansion wave structure, the use of refined grids to recover the same grid size after flow expansion is a good way of remedy, as can be seen from Fig. 9. Otherwise, with the application of ENO2 scheme, a great improvement in computational accuracy can be achieved, as is evident in Fig. 9.

The above examples illustrate the new procedure of generating streamline meshes as described in Step 4 which is different from that reported in [1]. The present procedure is an improvement over the work in [1], particularly when there exists a solid boundary. The streamline mesh generating procedure presented in [1] may cut into the solid body and causing smaller CFL number that can be uscd, i.c. smaller marching step sizc.

To apply the streamline Godunov method to more practical equilibrium gas dynamical flows, we next consider a case of a supersonic air flow past a $10 \%$ symmetric circular-arc airfoil with $M_{\infty}=8, P_{\infty}=101325 \mathrm{~N} /$ $\mathrm{m}^{2}, T_{\infty}=1000^{\circ} \mathrm{K}$ at zero incidence. Here, 1000 initial uniform cells are used with $\Delta y_{\text {ini }}=0.001$. The computed pressure contour and streamline grids for the equilibrium airflow are presented in Fig. 10. The leading-edge and trailing-edge oblique shock waves and the Mach waves emanating from the surface are all crisply captured. The gridlines clearly indicate also that the computational mesh follows exactly the particle movement even when it crosses a shock wave. An ENO2 scheme is used in this case. From the comparison between perfect and equilibrium flows for the surface distribution of the flow properties, as shown in Fig. 11, we know that the real gas effect should reduce the shock strength and also the shock angle in general.

Another example considered is that of shock interaction generated in a convergent channel containing both upper and lower wall slope discontinuities with $-10^{\circ}$ and $20^{\circ}$ wedge angles, respectively. The collision of the two shocks belonging to different families produces two new shocks and a slipline discontinuity. The exact solution to this case can be constructed using the boundary Riemann solver to predict the location and strengths 
(a)

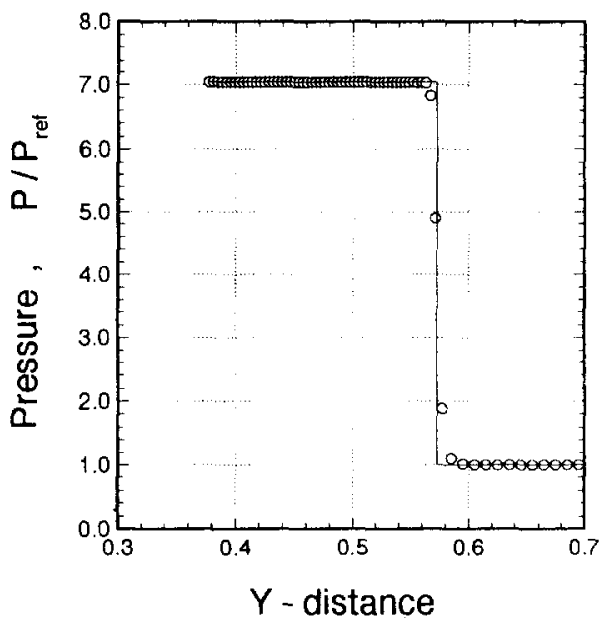

(c)

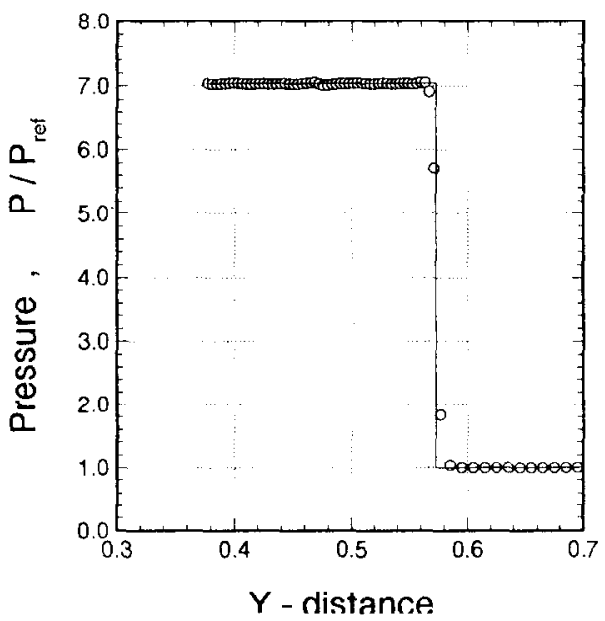

(b)

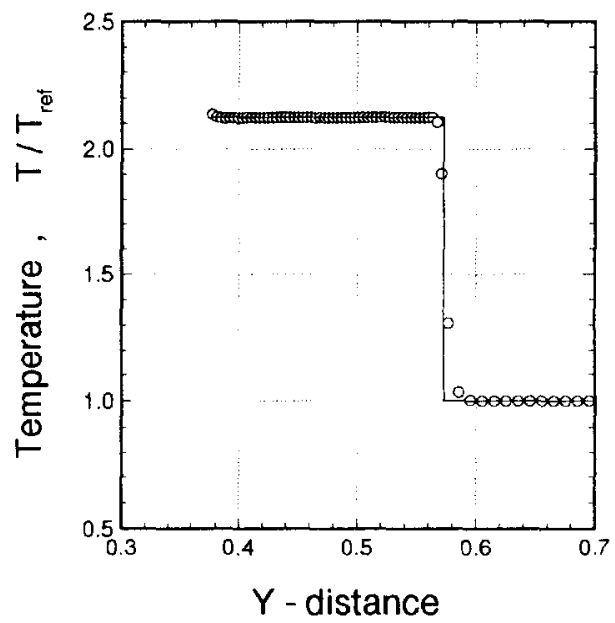

(d)

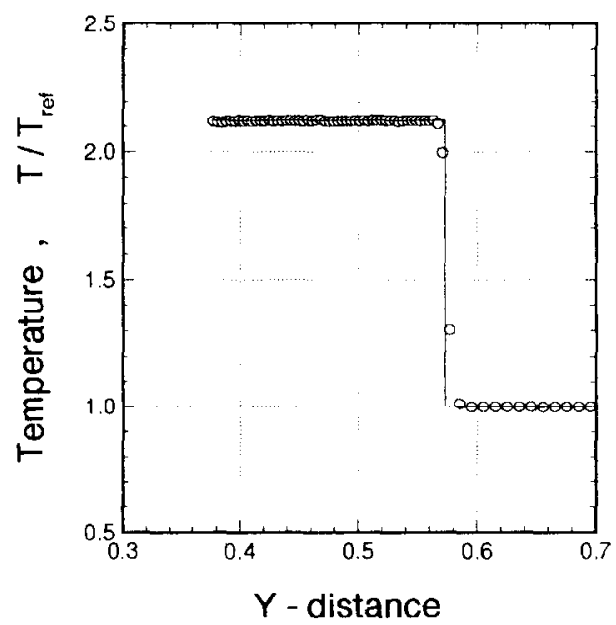

Fig. 8. Distribution of flow properties of a perfect wedge air flow along a marching distance line $(x=1.0)$. A comparison of numerical results (circles) and exact solution (solid linc). (a) and (b) First-order Godunov scheme: (c) and (d) second-order ENO schemre.

of the shocks induced by the upper and lower wall slope discontinuities. Fig. 12 illustrates the computed streamline grids for the perfect and equilibrium air flows with the same frecstream conditions and initial cell sizes as above, using ENO2 scheme. Flowfield structures accompanying with the shock - shock interaction system are clearly represented. The flow properties computed with both first order Godunov and second order ENO schemes are shown along with the exact solution in Fig. 13 at station $x=0.6$ which is upstream of the shock-shock interaction and in Fig. 14 at station $x=0.75$, which is downstream of the shock-shock interaction, respectively. Our numerical results agree well with the exact solutions, as can be seen from Figs. 13 and 14 . Obviously, the ENO2 results can achieve a better resolution for the shocks and slipline discontinuity, especially for the flow properties downstream of the shock-shock interaction.

The next example is that of interaction of shocks of the same family resulting from a supersonic air flow past 
(a)

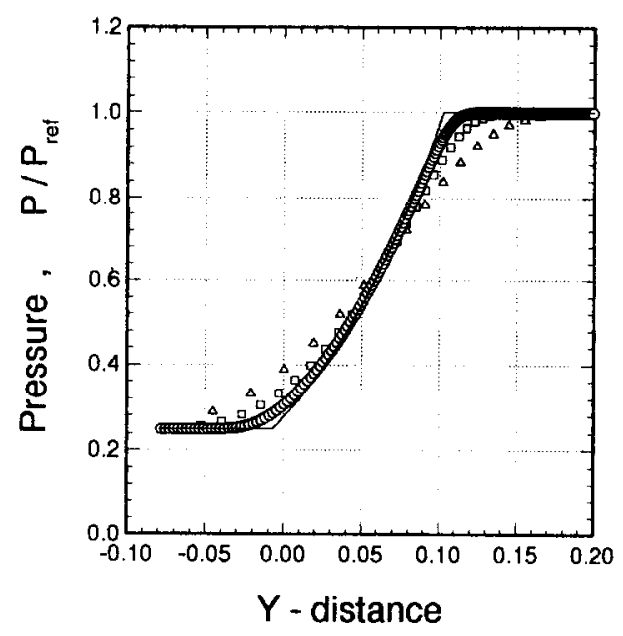

(c)

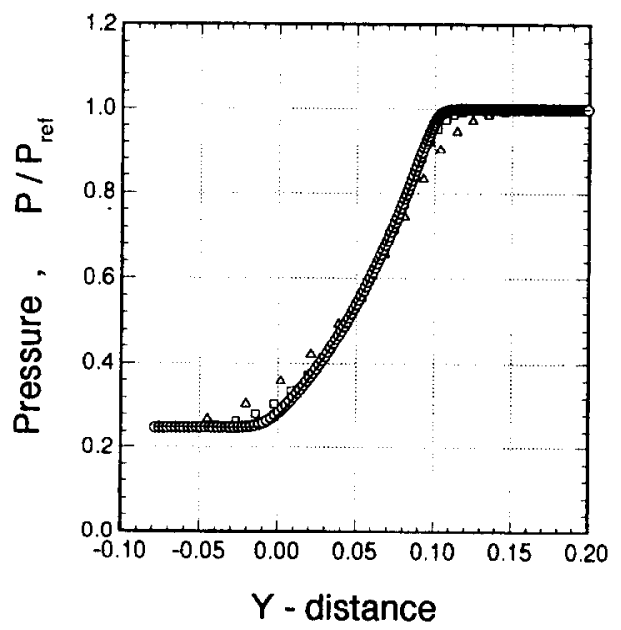

(b)

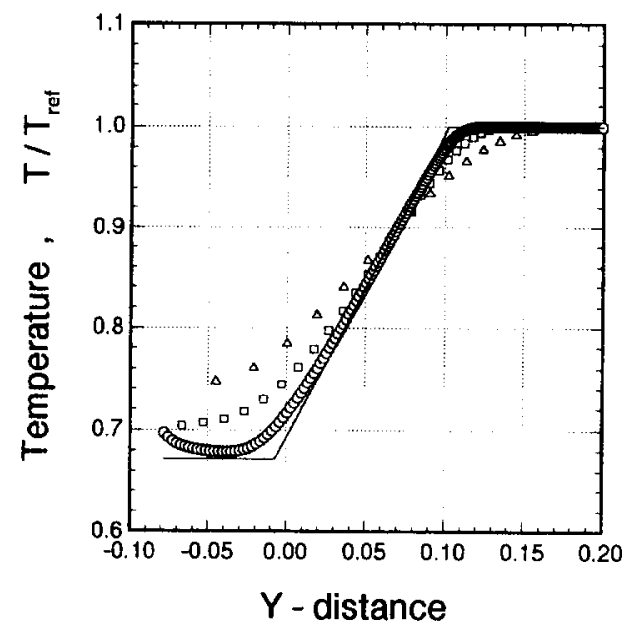

(d)

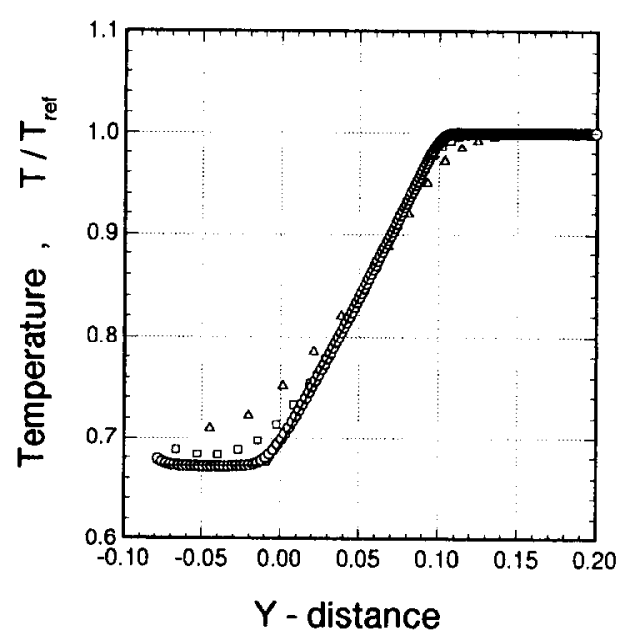

Fig. 9. Distribution of flow properties of a perfect Prandtl-Meyer air flow along a marching distance line ( $x=0.5)$. A comparison of numerical results for $\Delta x_{\text {ini }}=0.01$ (triangles), 0.005 (squares), 0.001 (circles), and exact solution (solid line). (a) and (b) First-order Godunov scheme; (c) and (d) second-order ENO scheme.

(a) Pressure Contour

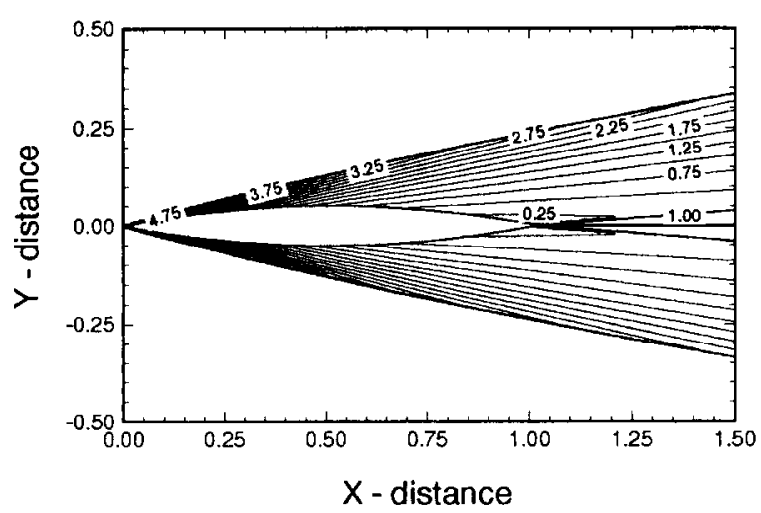

(b) Streamline Grids

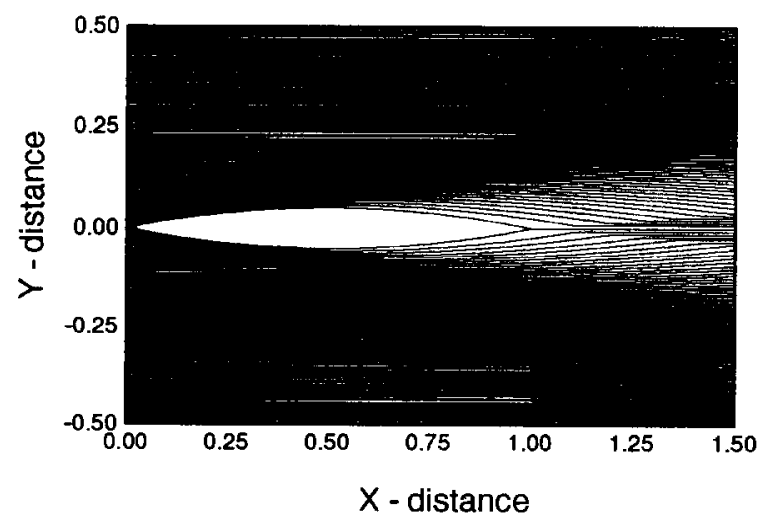

Fig. 10. Numerical results of a supersonic equilibrium air flow past a $10 \%$ symmetric circular-arc airfoil $\left(M_{\infty}=8\right)$ using the ENO2 scheme. (a) Pressure contour; (b) streamline grids. 
(a)

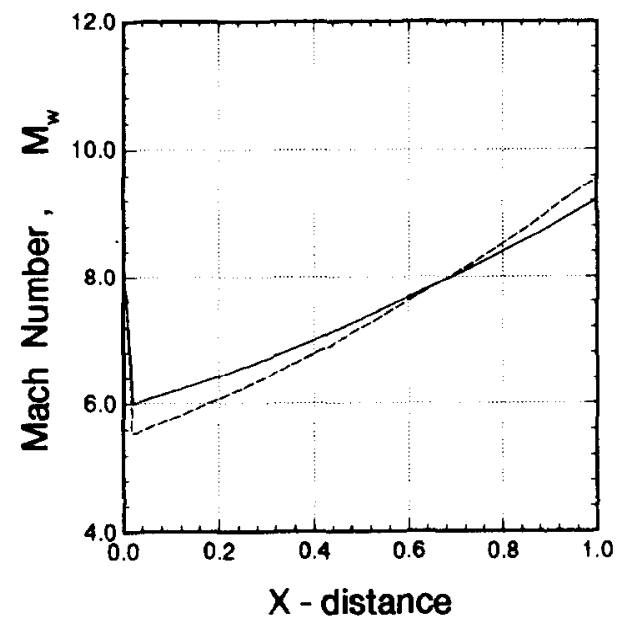

(c)

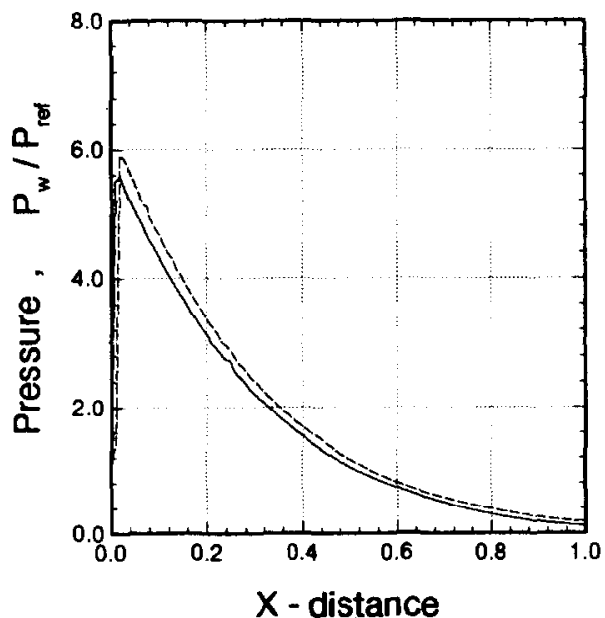

(b)

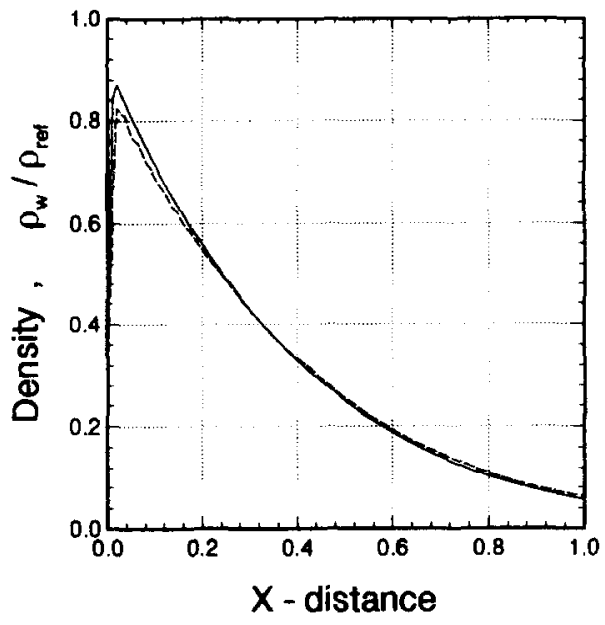

(d)

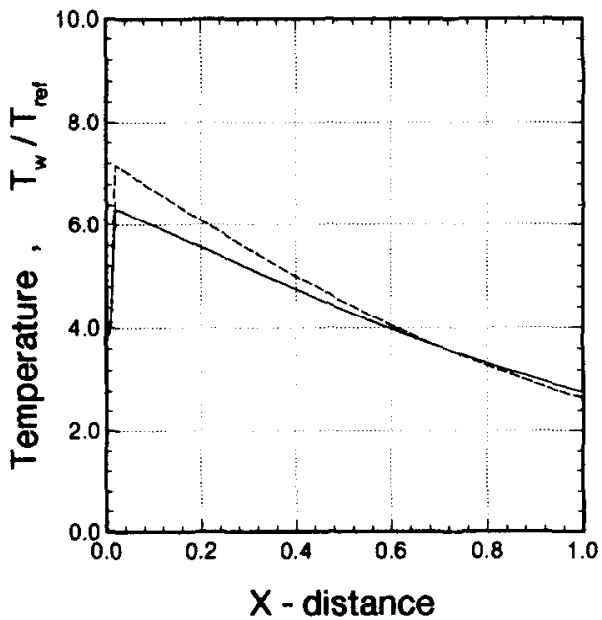

Fig. 11. Distribution of surface flow properties of a supersonic air flow past a $10 \%$ symmetric circular-arc airfoil using the ENO2 scheme. A cumparison of perfect flow (dash line) and cquilibrium flow (solid line). (a) Mach number; (b) density; (c) pressure; (d) temperature

(a) Perfect Air Flow

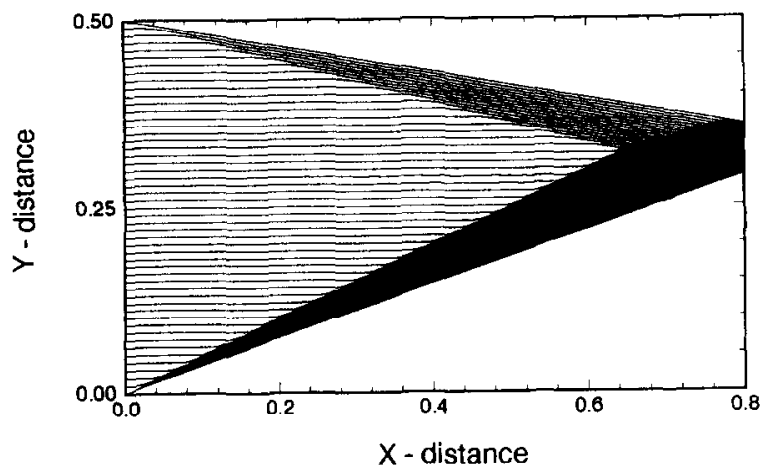

(b) Equilibrium Air Flow

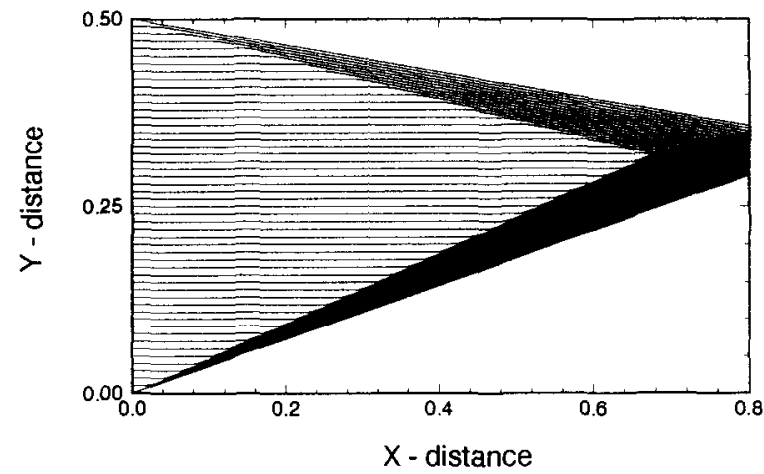

Fig. 12. Streamline grids of a supersonic air flow past a converging channel $\left(M_{x}=8\right)$ using the ENO2 scheme. (a) Perfect flow; (b) equilibrium flow. 
a double-wedge with $10^{\circ}$ and $20^{\circ}$ wedge angles for both perfect and equilibrium air. All the computational conditions are the same as the prcvious cxample. The cxact solution consists of three parts: the oblique shock solutions for the first and second wedges and the shock collision formed by the freestream flow and the uniform flow behind the second shock. The exact solutions for the first and second oblique shocks can be obtained using the boundary Riemann solver and then the shock-shock collision is solved exactly by the real gas Riemann solver. Since the two colliding shocks are from the same family, the resulting slipline inclination (about $21.5^{\circ}$ ) is very close to that of the uniform flow behind the second shock $\left(20^{\circ}\right)$. This implies that the turning angle in the Prandtl-Meyer expansion is small and the expansion fan becomes very narrow. The computed pressure contours and streamline grids are shown in Fig. 15 to illustrate these phenomena. From the results of the flow properties along the solid wall, as shown in Fig. 16, we know that except for the strengths of the shocks, the flow structure accompanying with the shocks and reflection expansion wave are not significantly different for the perfect and

(a)

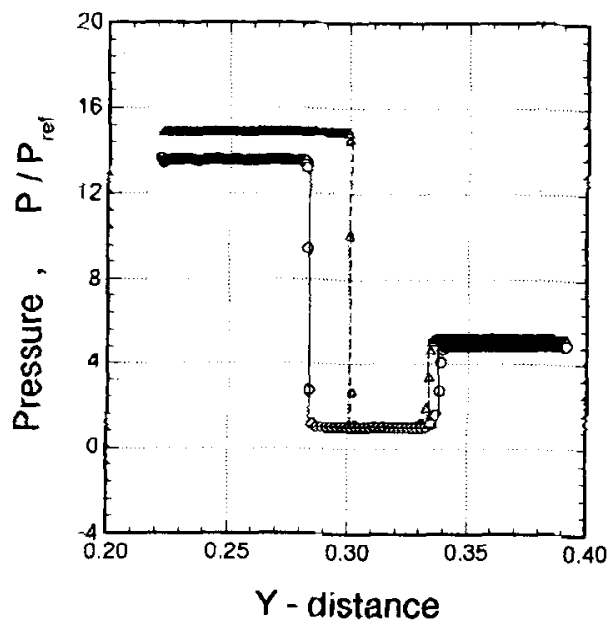

(c)

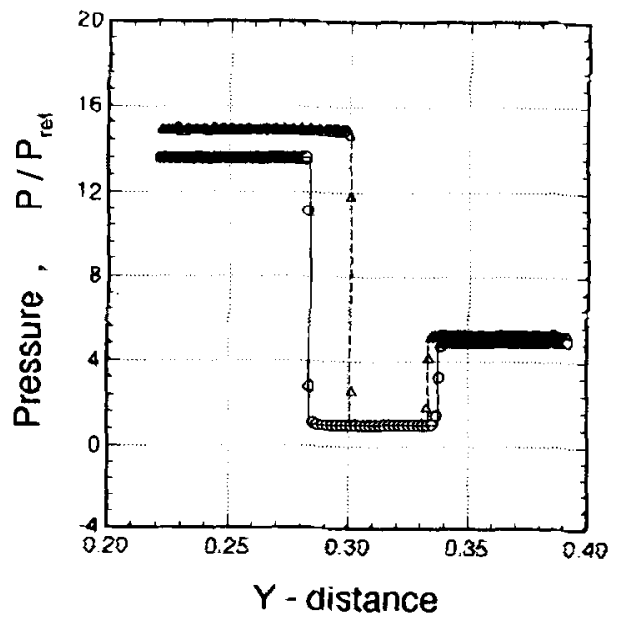

(b)

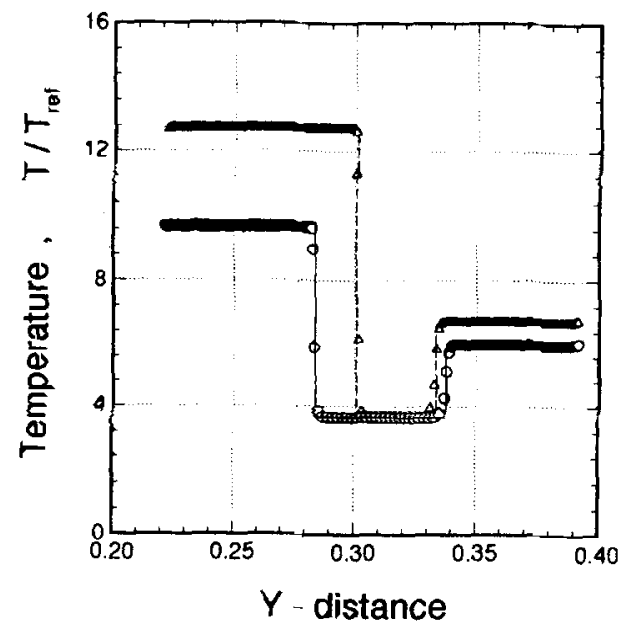

(d)

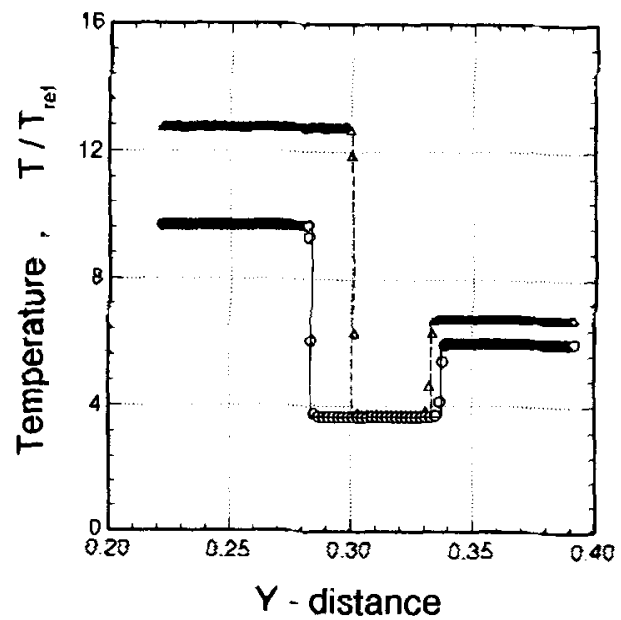

Fig. 13. Distribution of flow properties of a supersonic air flow past a converging channel along a marching distance line $(x=0.6)$. A comparison of numerical results for perfect flow (triangles) and equilibrium flow (circles) and exact solution for perfect flow (dash line) and equilibrium flow (solid line). (a) and (b) First-order Godunov scheme; (c) and (d) second-order ENO scheme. 
(a)

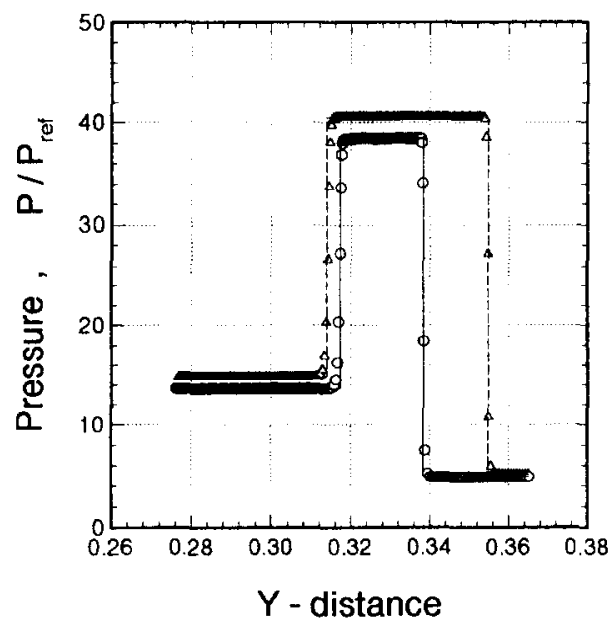

(c)

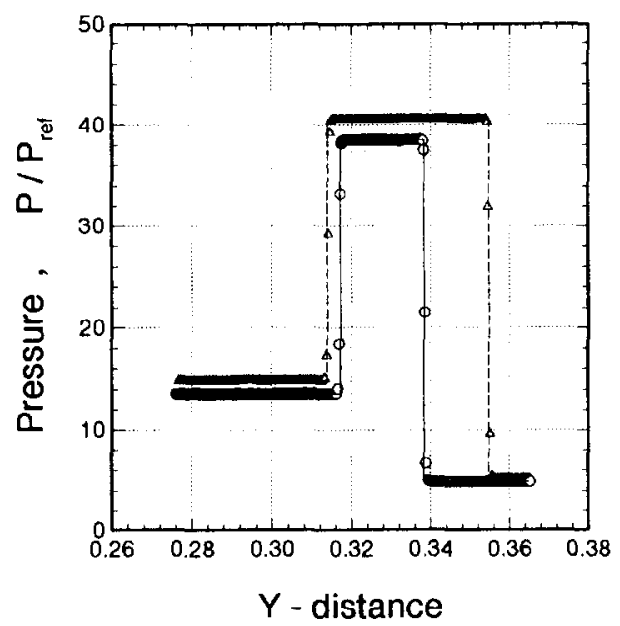

(b)

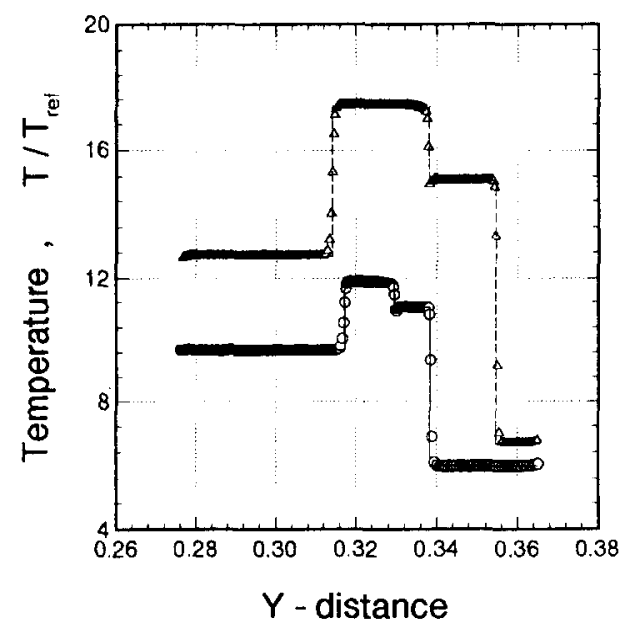

(d)

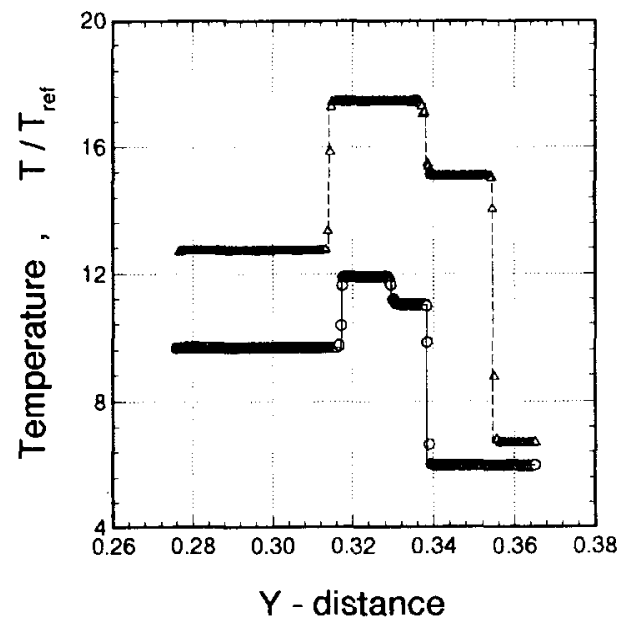

Fig. 14. Same as in Fig. 13, properties are plotted along a marching distance line $(x=0.75)$. A comparison of numerical results for perfect now (triangles) and equilibrium flow (circles) and exact solution for perfect flow (dash line) and equilibrium flow (solid line). (a) and (b) First-order Godunov scheme; (c) and (d) second-order ENO scheme.

(a) Pressure Contour

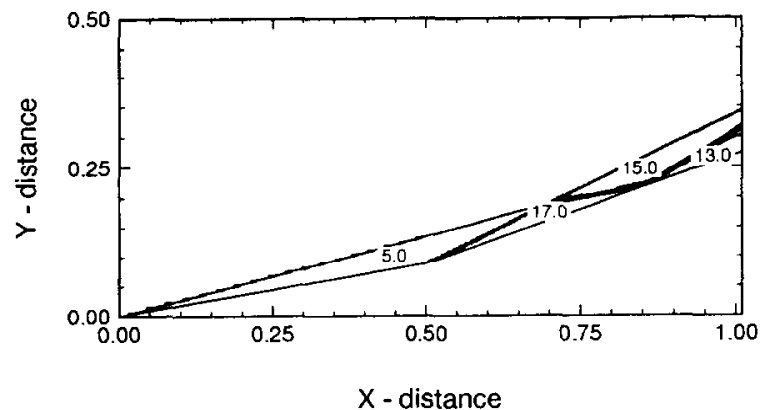

(b) Streamline Grids

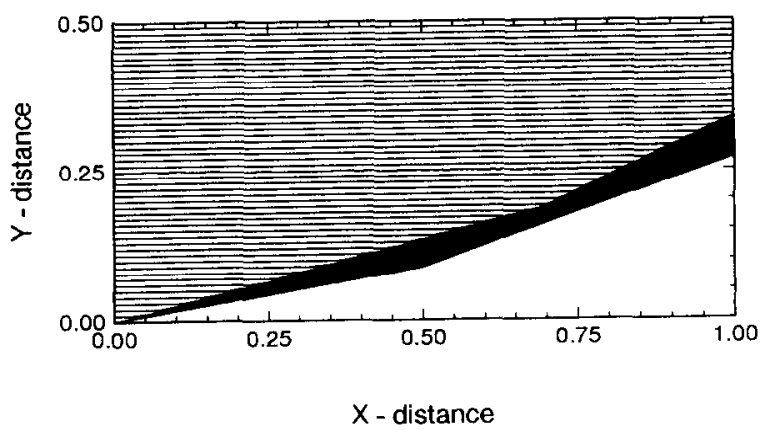

Fig. 15. Numerical results of a supersonic equilibrium air flow past a double-wedge $\left(M_{x}-8\right)$ using the ENO2 scheme. (a) Pressure contour; (b) streamline grids. 
(a)

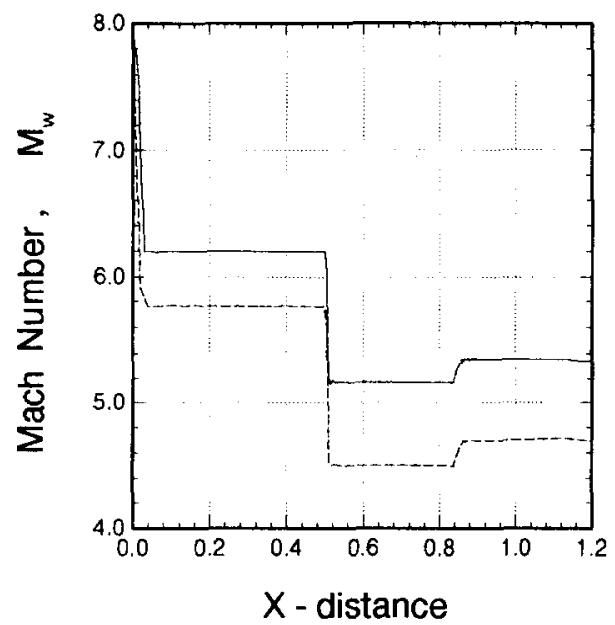

(c)

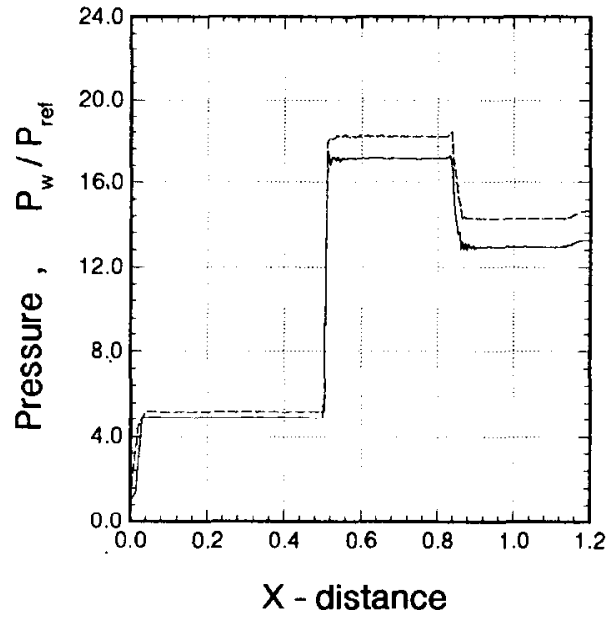

(b)

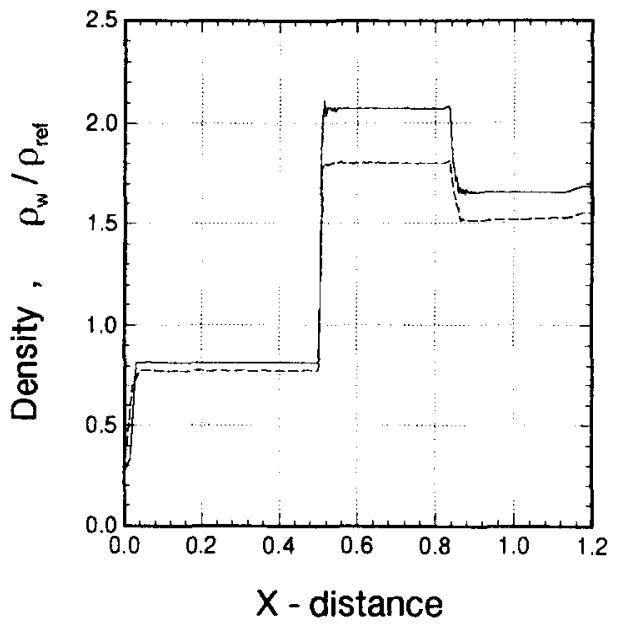

(d)

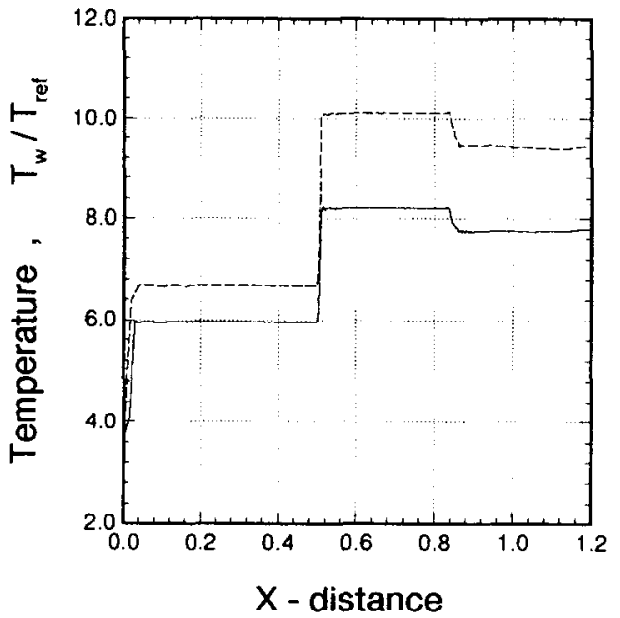

Fig. 16. Distribution of surface flow properties of a supersonic air flow past a double-wedge using the ENO2 scheme. A comparison of perfect flow (dash line) and equilibrium flow (solid line). (a) Mach number; (b) density; (c) pressure; (d) temperature.

(a) Pressure Contour

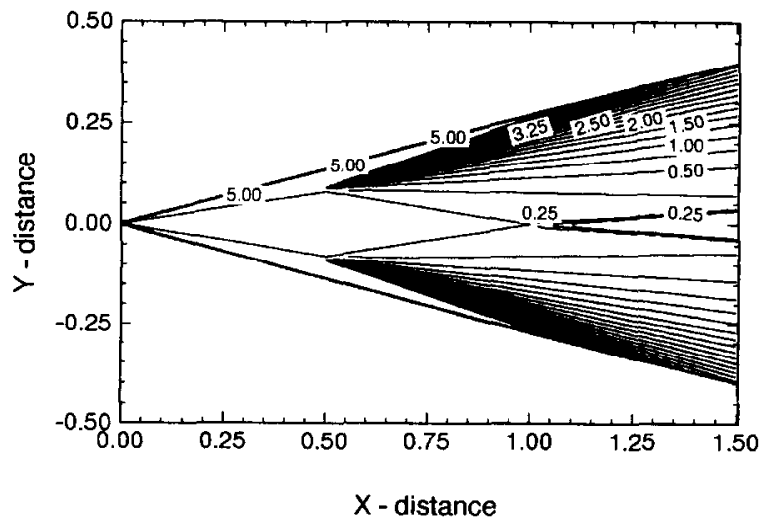

(b) Streamline Grids

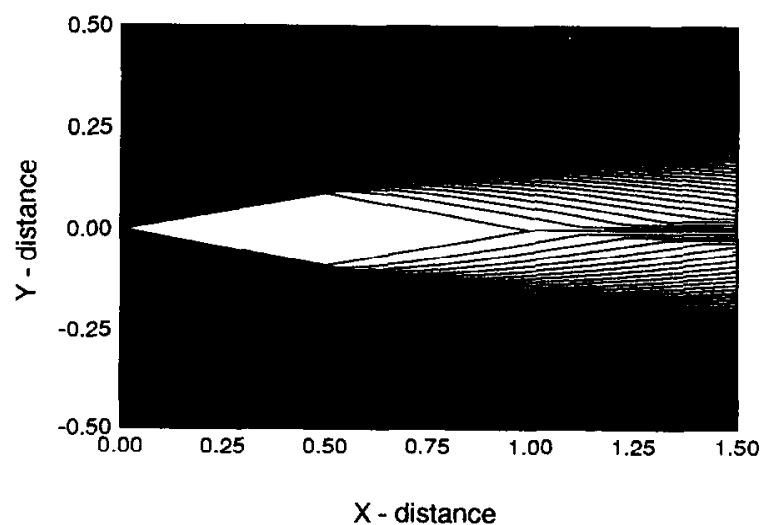

Fig. 17. Numerical results of a supersonic equilibrium air flow past a diamond-shaped airfoil $\left(M_{\infty}=8\right)$ using the ENO2 scheme. (a) Pressure contour; (b) streamline grids. 
equilibrium air flows in this case. It is also seen from Fig. 16 that the strength of expansion wave is still strong enough to generate a greater decrease in the flow propertics on the solid wall.

We also consider a supersonic air flow past a diamond-shaped airfoil with a $10^{\circ}$ wedge angle. The freestream conditions and initial cell size are the same as the previous example for both the perfect and equilibrium air flows. The computed pressure contours and streamline grids for the equilibrium airflow are presented in Fig. 17. The leading-edge and trailing-edge oblique shock waves and the Prandtl-Meyer expansion fan at the vertex are all cleanly and accurately resolved. The distribution of surface flow properties for the perfect and equilibrium flows, as shown in Fig. 18, indicate that the real gas effect should weaken the shock strength and result lower temperature and higher Mach number after the leading-edge shock as compared with that for a perfect gas. After the Prandtl-Meyer expansion at the vertex, higher temperature and lower Mach number are obtained for equilibrium gas, as the conversion of internal energy to kinetic energy is easier and more fully for perfect gas. Note that the poor accuracy occurring at the expansion side of flowfield after the flow across the vertex of airfoil

(a)

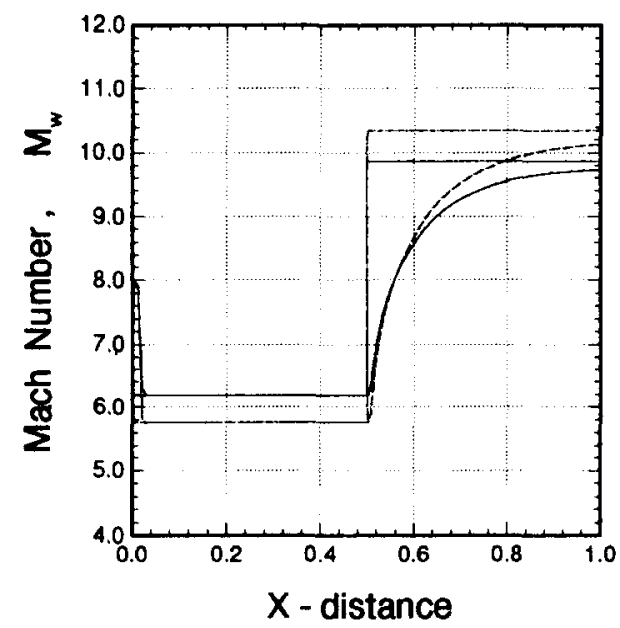

(c)

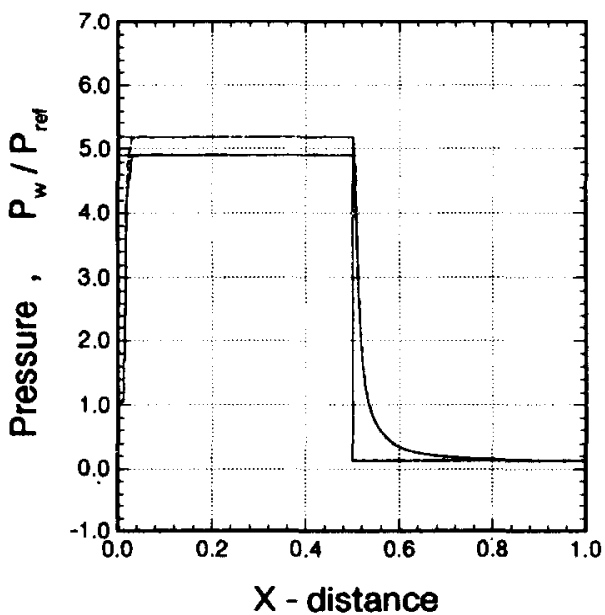

(b)

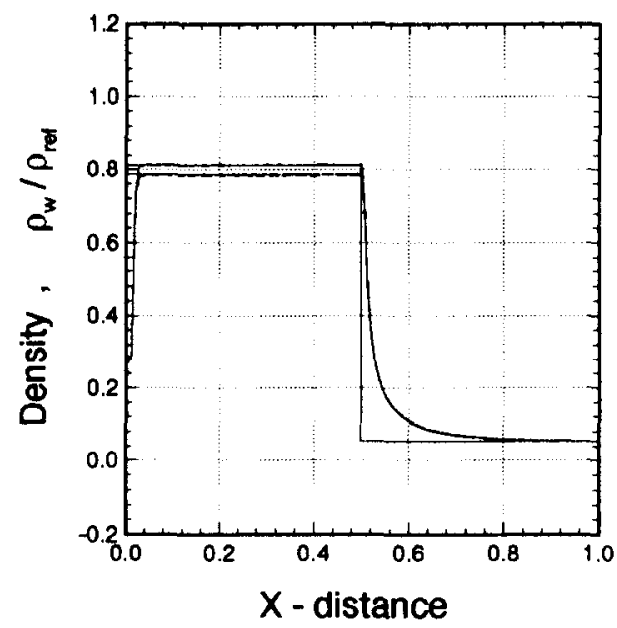

(d)

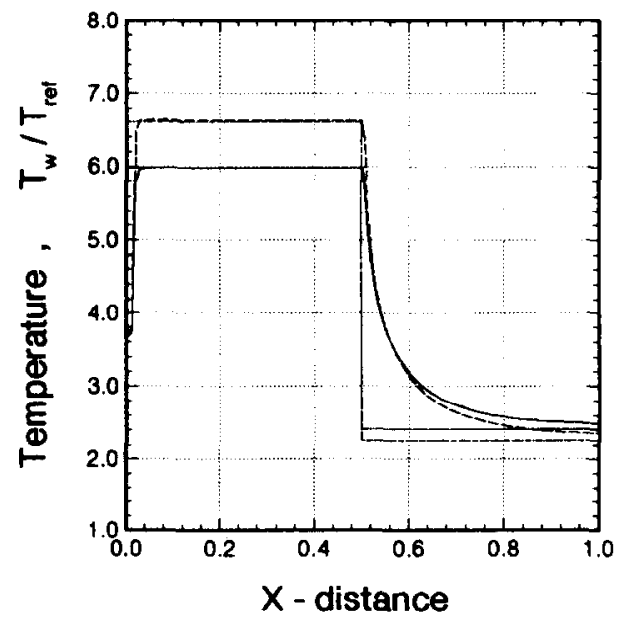

Fig. 18. Distribution of surface flow properties of a supersonic air flow past a diamond-shaped airfoil using the ENO2 scheme. A comparison of perfect flow (dash line) and equilibrium flow (solid line). (a) Mach number; (b) density; (c) pressure; (d) temperature. 
(a) Perfect Air Flow

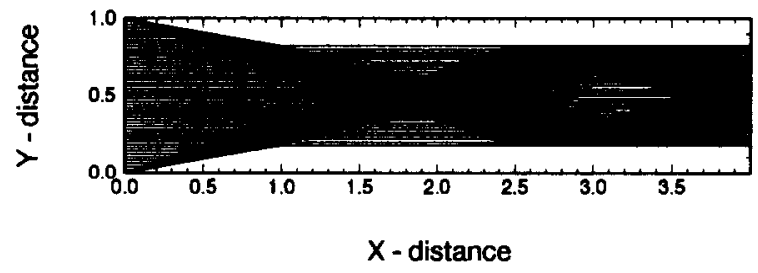

(b) Equilibrium Air Flow

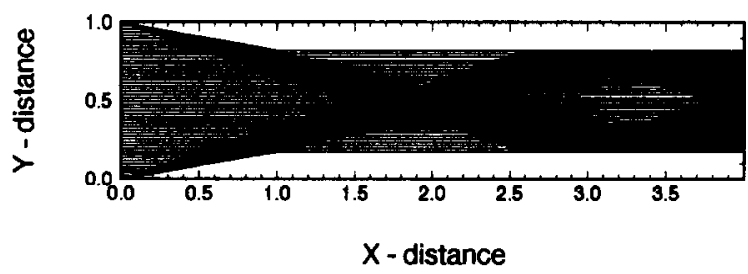

(c) Equilibrium IDG-O $\mathrm{O}_{2}$ Flow

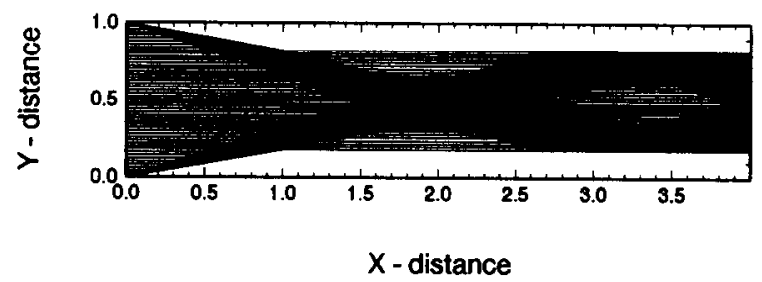

Fig. 19. Streamline grids of a high-temperature channel flow ( $\left.M_{\infty}-5\right)$ using the ENO2 scheme. (a) Pcrfect air flow; (b) cquilibrium air flow; (c) equilibrium IDG-O, flow.

comes from the fact that the streamlines delay to match the solid wall due to the existence of the sudden expansion corner. By refining the cell height at the initial marching step near the solid wall one could achieve a better accuracy.

To test different equilibrium gas models we consider a high-temperature channel flow through a simple inlet which consists of a $10^{\circ}$ wedge followed by a $10^{\circ}$ expansion shoulder and then connected by a constant-area duct. At the inlet entrance, all conditions are held constant at the reference freestream conditions of $M_{\infty}=5$, $P_{\infty}=101325 \mathrm{~N} / \mathrm{m}^{\prime}$ and $T_{\infty}=3576^{\circ} \mathrm{K}$. We have computed this problem using both the Tannehill's equilibrium air table and Lighthill's IDG- $\mathrm{O}_{2}$ gas model. Here, 500 initial uniform cells are used with $\Delta y_{\text {ini }}=0.002$. The computed streamline grids of the perfect and equilibrium flows for these two gas models are presented in Fig. 19. And the pressure and temperature contours are shown in Figs. 20 and 21 for these two equilibrium gas

(a) Perfect Air Flow

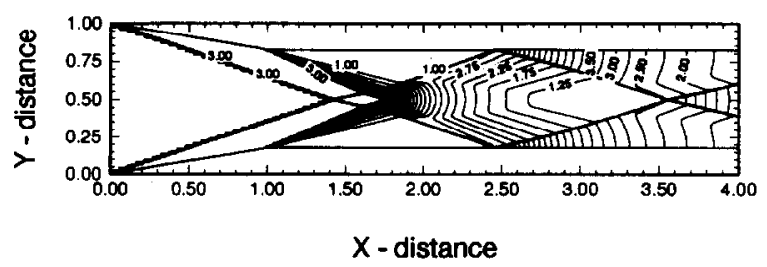

(b) Equilibrium Air Flow

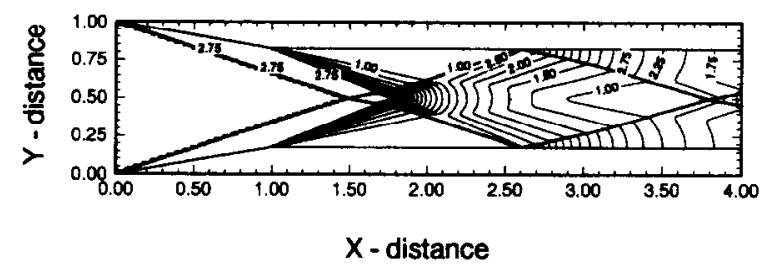

(c) Equilibrium IDG-O $\mathrm{O}_{2}$ Flow

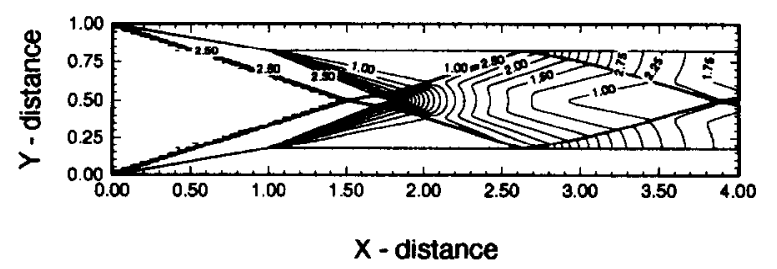

Fig. 20. Pressure contours of a high-temperature channel flow $\left(M_{\infty}=5\right.$ ) using the ENO2 scheme. (a) Perfect air flow; (b) equilibrium air flow; (c) equilibrium IDG-O ${ }_{2}$ flow. 
(a) Perfect Air Flow

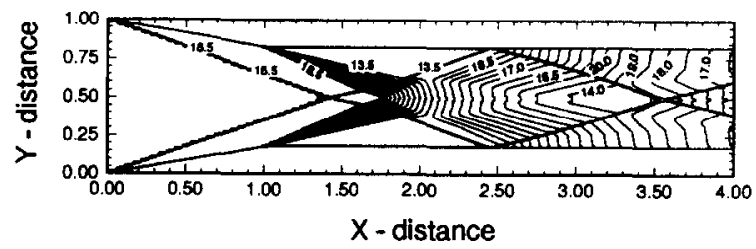

(b) Equilibrium Air Flow

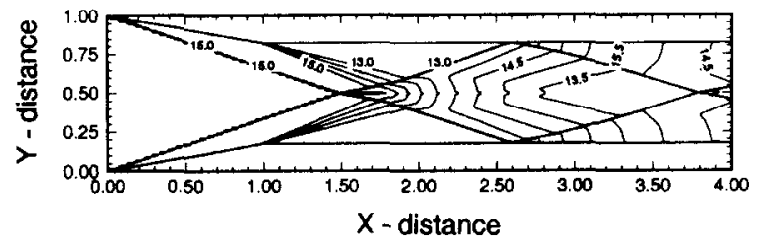

(c) Equilibrium IDG-O $\mathrm{O}_{2}$ Flow

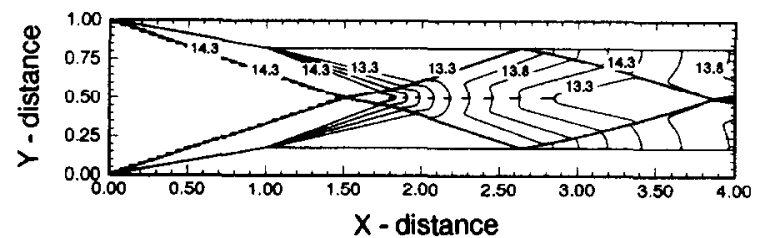

Fig. 21. Temperature contours of a high-temperature channel flow $\left(M_{\%}=5\right)$ using the ENO2 scheme. (a) Perfect air flow; (b) equilibrium air flow; (c) equilibrium IDG-O $\mathrm{O}_{2}$ flow.

(a)

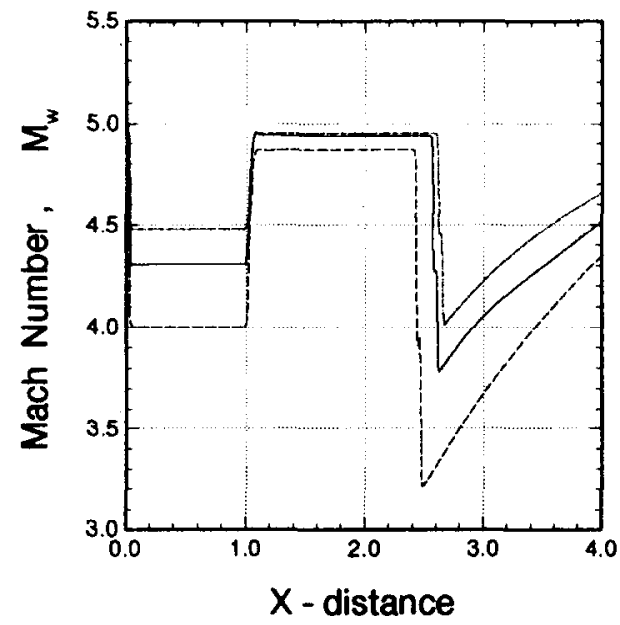

(c)

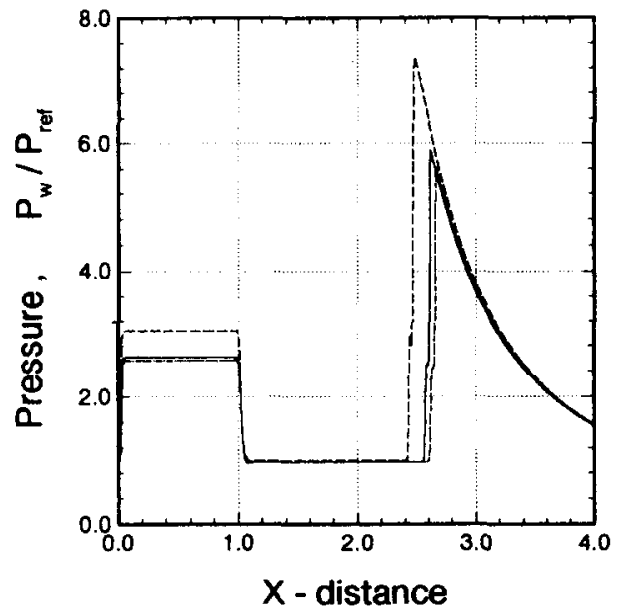

(b)

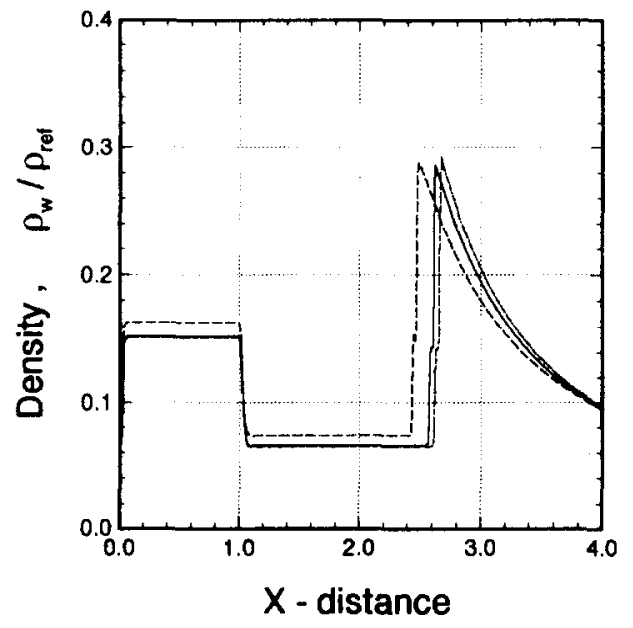

(d)

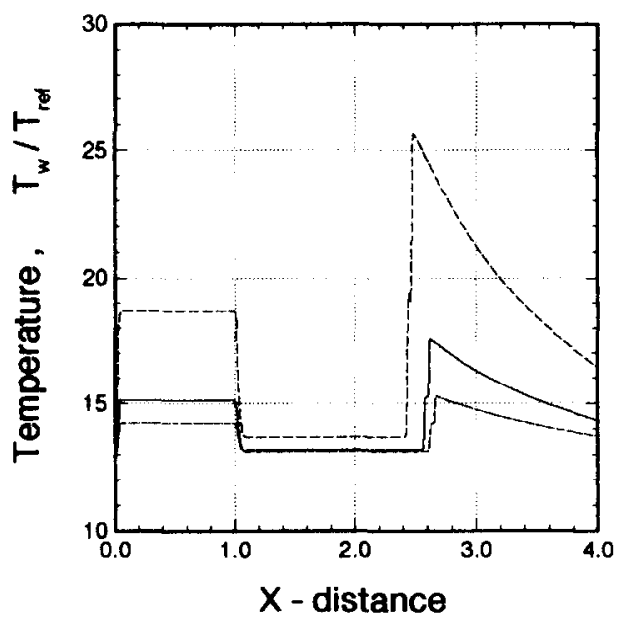

Fig. 22. Distribution of surface flow properties for a high-temperature channel flow using the ENO2 scheme. A comparison of perfect air flow (dash line), equilibrium air flow (solid line) and equilibrium IDG-O flow (dash-dot line). (a) Mach number; (b) density; (c) pressure; (d) temperature. 
models, respectively. It can be clearly seen that the shock strength is smaller for the real gas and the values of thermodynamic properties are reduced behind the shocks, since the kinetic energy would attribute to the internal degree of freedom for the equilibrium flow. Comparing the results in Figs. 20 and 21, it is quite surprising to find the close matching of the values for the field distribution of the pressure and temperature between these two equilibrium gas models. In Fig. 22, the distributions of the surface flow properties indicate the effects of the real gas and the difference of the two equilibrium gas models. The distributions of the flow properties along centerline are also shown in Fig. 23. Aside from the underestimation in temperature and overestimation in Mach number along the solid wall and centerline, the overall good agreement for pressure and density in the equilibrium condition shows that the Lighthill's IDG model is a valid model for the numerical computation of equilibrium air flows. The underestimation in temperature for the Lighthill's IDG model attributes to the fact that this model may overestimate the dissociation degree of molecules in the mixture at temperature above about

(a)

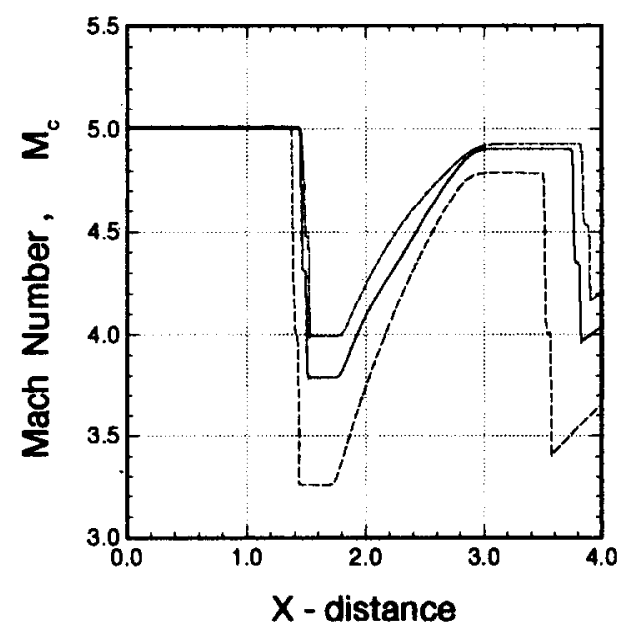

(c)

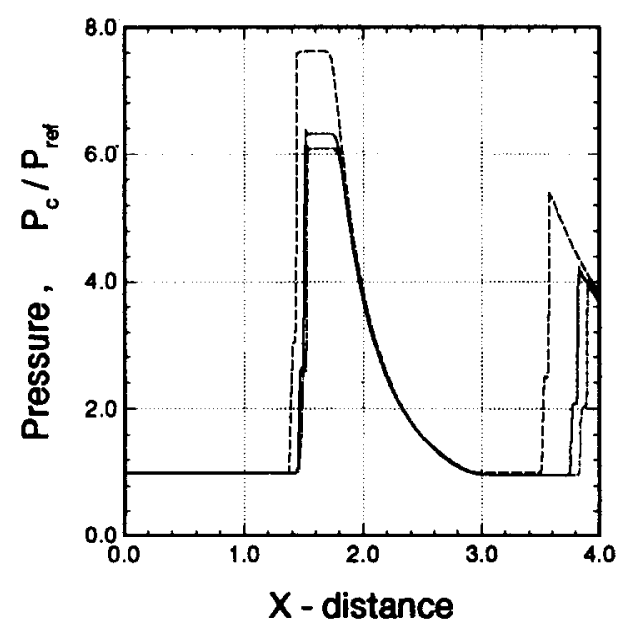

(b)

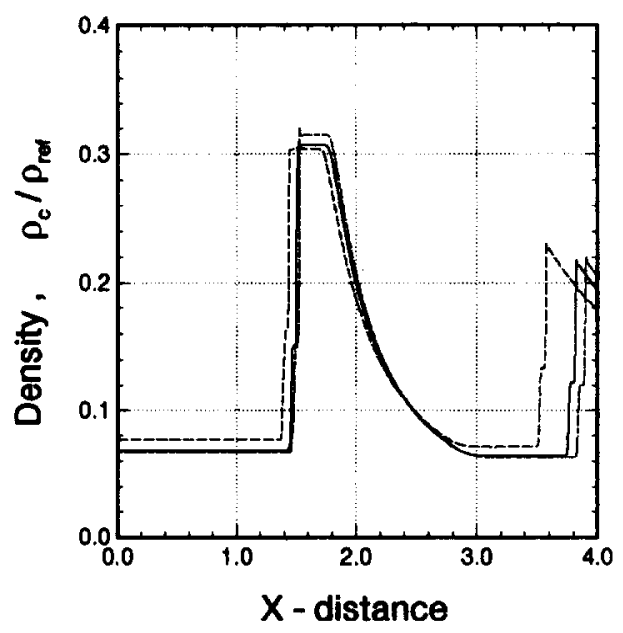

(d)

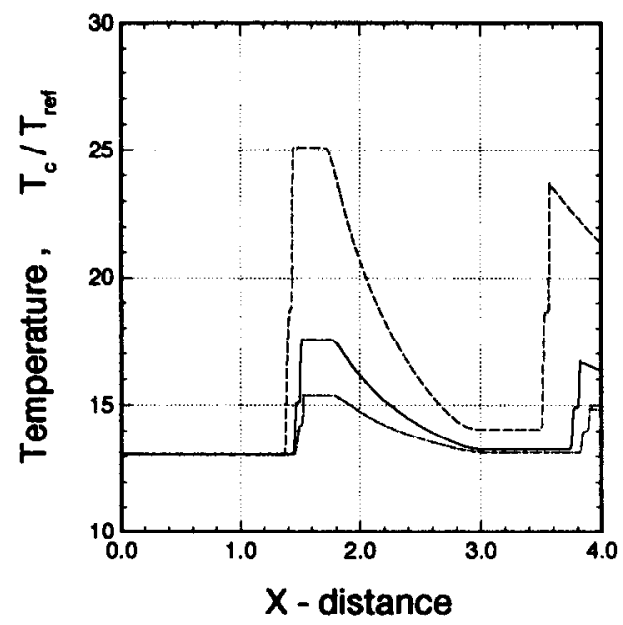

Fig. 23. Distribution of flow properties along centerline for a high-temperature channel flow using the ENO2 scheme. A comparison of perfect air flow (dash line), equilibrium air flow (solid line) and equilibrium IDG-O ${ }_{2}$ flow (dash-dot line). (a) Mach number; (b) density; (c) pressure; (d) temperaturc. 
$3500^{\circ} \mathrm{K}$. Therefore, if more internal energy contributes from the dissociation energy of chemical reaction, and then the higher the temperature the more underestimation in temperature may result, as are seen from Figs. 22 and 23. Generally speaking, we can use Lighthill's IDG-O model as a simple, useful equilibrium gas model to the approximate numerical simulation of the flowfield properties for a chemically equilibrium reacting air.

To evaluate the robustness of the splitting technique for the system Eq. (1) involving source terms in our streamline Godunov method, we consider here a hypersonic flow past an axisymmetrical circular cone with $20^{\circ}$ cone angle with $M_{\infty}=5, P_{\infty}=101325 \mathrm{~N} / \mathrm{m}^{2}, T_{\infty}=3576^{\circ} \mathrm{K}$ at zero incidence. This is the Taylor-Maccoll flow in an equilibrium gas. Both the Tannehill's air table and Lighthill's IDG model are used. Here, we use 100 cells and $\Delta y_{\text {ini }}=0.005$. Computed results for the flow properties at section $x=0.6$, obtained using both first-order Godunov scheme and ENO2 scheme, are shown in Fig. 24 along with the exact solution which was obtained by solving the Taylor-Maccoll equation for equilibrium gases. Very good agreement is found between the computation and exact solution. The ENO2 results indicate better resolution at shock.

(a)

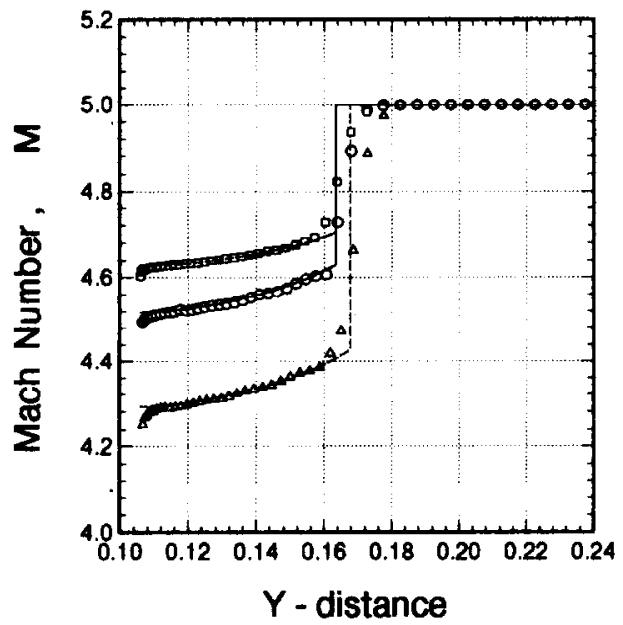

(c)

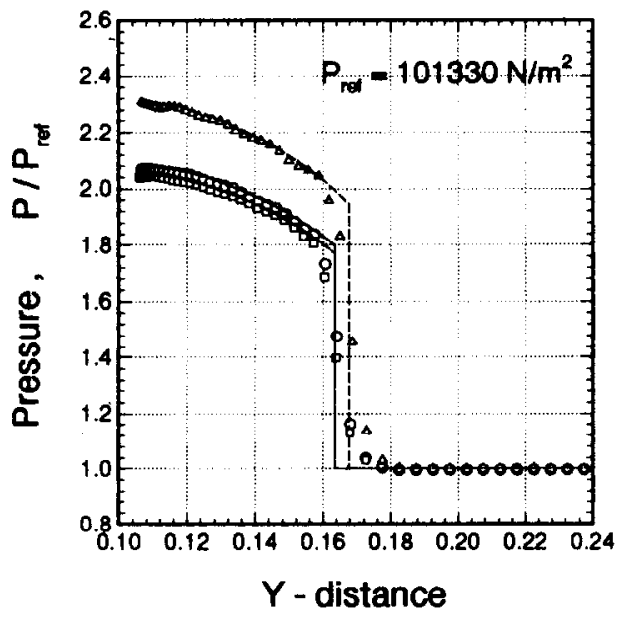

(b)

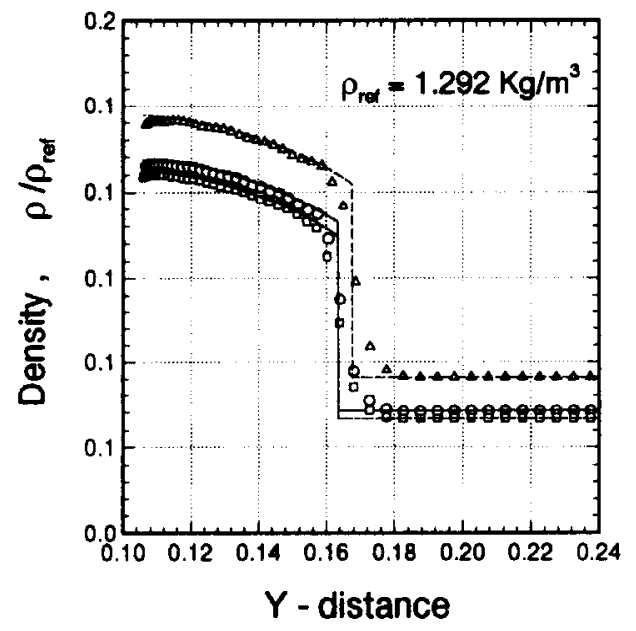

(d)

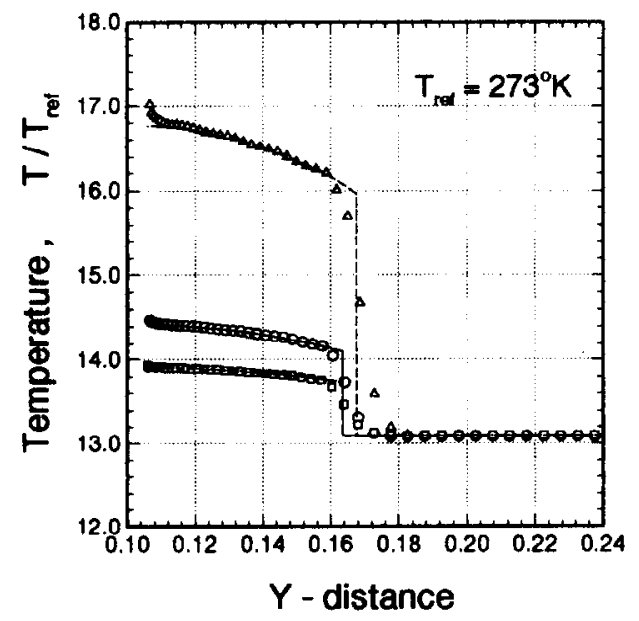

Fig. 24. Distribution of flow properties of a hypersonic Taylor-Maccoll flow along a marching distance line $x=0.6\left(M_{\times}=5\right)$ using the ENO2 scheme. A comparison of numerical results for perfect air flow (triangles), equilibrium air flow (circles), and equilibrium IDG-O 2 flow (squares) with their corresponding exact solution. (a) Mach number; (b) density; (c) pressure; (d) temperature. 


\section{Concluding remarks}

In this paper, a Godunov-type method with streamline meshes as control volumes is presented for solving the steady supersonic and hypersonic equilibrium flows. The equilibrium real gas Riemann problem and its solution in the streamline mesh system is devised for the implementation of Godunov method. Two real gas models are adopted to calculate the equilibrium gas properties; one is Tannehill's program and the other is Lighthill's simplified ideal dissociating gas model. A two-step essentially non-oscillatory method is implemented to achieve high-order accuracy. Computations of two-dimensional and three-dimensional axisymmetrical steady supersonic and hypersonic equilibrium flows have been carried out and the results indicate that the smooth flow can be accurately represented and flow discontinuities such as oblique shock waves and sliplines can be crisply resolved. The use of streamline meshes as computational cells enables intrinsic flow adaption, excellent resolution of sliplines, and simpler implementation of the Godunov method. The use of Lighthill's simplified real gas model gives similar results as that given by Tannehill's program. It shows that this IDG model is not only a good quantitative representation of $\mathrm{O}_{2}$ and $\mathrm{N}_{2}$ alone, but also provides a good qualitative representation of air, even when NO is formed. Formula in the Lighthill's model are all explicit for different kinds of equilibrium diatomic gas, and this IDG model is very efficient to extend our streamline Godunov method to the numcrical computation of chemical nonequilibrium flow. The computational algorithm is robust and accurate and is very suitable for steady supersonic and hypersonic equilibrium flow calculations. Extension of the present streamline Godunov-type scheme to steady supersonic and hypersonic nonequilibrium flows is a subject of future study.

\section{Acknowledgment}

This work was sponsored by the National Science Council of the Republic of China under Grant NSC 86-2612-E002-010.

\section{References}

[1] C.A. Hsu and J.Y. Yang, A high order streamline Godunov scheme for steady supersonic flow computation, J. Comput. Methods Appl. Mech. Engrg. (June, 1993).

[2] S.K. Godunov, A difference method for numerical calculation of discontinuous solutions in hydrodynamics, Mathematicheskii Sbornik 47 (1957) 271-306.

[3] P. Colella and H.M. Glaz, Efficient solution algorithms for the Riemann problem of real gases, J. Comput. Phys. 59 (1985) $264-289$.

[4] P. Gleister, An approximate linearized Riemann solver for the Euler equations for real gases, J. Comput. Phys. 74 (1988) $382-408$.

[5] B. Grossman and R.W. Walters, Analysis of flux-split algorithms for Euler's equations with real gases, AIAA J. 27(5) (1989) 524-531.

[6] J.L. Montagné, H.C. Yee and M. Vinokur, Comparative study of high-resolution shock-capturing schemes for a real gas, AIAA J. 27(10) (1989) 1332-1346

[7] A. Suresh and M.S. Liou, Osher's scheme for real gases, AIAA J. 29(6) (1991) 920-926.

[8] M. Vinokur and J.L. Montagné, Generalized flux-vector splitting and Roe average for an equilibrium real gas, J. Comput. Phys. 89 (1990) 276-300.

[9] S. Srinivasan, J.C. Tannehill and K.J. Weilmunster, Simplificd curve fit for the thermodynamic properties of equilibrium air, NASA Reference Publication 1181, August 1987.

[10] M.J. Lighthill, Dynamics of a dissociating gas. Part I. Equilibrium flow, J. Fluid Mech. 2(1) (1957) 1-12.

[11] M. Vinokur and Y. Liu, Equilibrium gas flow computation II. An analysis of numerical formulations of conservation laws, AIAA Paper 88-0127, January 1988.

[12] J.L. Steger and R.F. Warming, Flux vector splitting of inviscid gas dynamic equations with application to finite difference methods, J. Comput. Phys. 40 (1981) 263-293.

[13] B. Van Leer, Flux-vector splitting for the Euler equations, ICASE Report 82-30, September 1982.

[14] P.L. Roe, Approximate Riemann solvers, parameter vectors, and difference schemes, J. Comput. Phys. 43 (1981) $357-372$.

[15] H.M. Glaz and A.B. Wardlaw, A high-order Godunov scheme for steady supersonic gas dynamics, J. Comput. Phys. 58 (1985) $157-187$.

[16] W.G. Vincenti and C.H. Kniger, Ir., Introduction to Physical Gas Dynamics 1st edition (John Wiley and Sons, Inc., New York, 1965).

[17] C.Y. Loh and W.H. Hui, A new Lagrangian method for steady supersonic flow computation. I. Godunov scheme, J. Comput. Phys. 89 (1990) 207-240.

[18] B. Van Leer, Towards the ultimate conservative difference scheme, V. A sccond order sequel to Godunov's method, J. Comput. Phys. 32 (1979) 101-136.

[19] J.Y. Yang, C.A. Hsu and W.H. Hui, Solution of the steady Euler equations in generalized Lagrangian formulation, AIAA J. 31(2) (1993) $266-272$.

[20] A. Harten and S. Osher, Uniformly second-order essentially nonoscillatory schemes, I., SIAM J. Numer. Anal. 24(2) (1987) 279-309. 